\title{
Copepod assemblages as a bioindicator of environmental quality in three coastal areas under contrasted anthropogenic inputs (Gulf of Gabes, Tunisia)
}

\author{
ZAHER DRIRA $^{1}$, SALMA KMIHA-MEGDICHE ${ }^{1}$, HOUDA SAHNOUN ${ }^{2}$, MARC TEDETTI $^{3}$, MARC PAGANO $^{3}$ \\ AND HABIB AYADI ${ }^{1}$ \\ ${ }^{1}$ Department of Life Sciences, Sfax Faculty of Sciences, Biodiversity and Aquatic Ecosystems UR/11ES72 Research Unit, University of \\ Sfax, Soukra Road Km 3.5. BP 1171 - PO Box 3000 Sfax, Tunisia, ${ }^{2}$ Coastal and Urban Environment Unit, IPEIS, P.C. '300o' Sfax, \\ Tunisia, ${ }^{3}$ Aix Marseille University, CNRS/INSU, University of Toulon, IRD, Mediterranean Institute of Oceanography (MIO) UM \\ 110, 13288 Marseille, France
}

\begin{abstract}
The study of copepod assemblages indicated the presence of 22 species and 12 families in the southern coast of Sfax, 20 species and 13 families in the northern coast and 14 species and 8 families in the Ghannouch area, with a dominance of Oithonidae (79, 51 and 43\% in the southern, northern and Ghannouch coasts, respectively). The relative abundance and the richness diversity of Oithonidae were found to be the most relevant indicators of anthropogenic pollution. Oithona nana, Euterpina acutifrons and Acartia clausi differed significantly in abundance between these three areas under differing degrees of pollution. The study of the structure, composition and density of the copepod fauna showed that the southern coast was a pollution-resistant ecosystem $\left(H^{\prime}=1.49 \pm 0.33\right.$ bits $^{-1} d^{-1} ; 22$ species; density $=51.375 \pm 4.340 \times$ $10^{3}$ ind $\left.\mathrm{m}^{-3}\right)$ followed by Ghannouch area $\left(H^{\prime}=1.74 \pm 0.28\right.$ bits ind $^{-1} ; 15$ species; density $=11.979 \pm 5.651 \times$ $10^{3}$ ind $\left.\mathrm{m}^{-3}\right)$ and the northern coast, considered as a restored area $\left(H^{\prime}=1.95 \pm 0.26\right.$ bits ind ${ }^{-1} ; 21$ species; density $=$ $6.516 \pm 4.304 \times 10^{3}$ ind $\left.\mathrm{m}^{-3}\right)$. The three ecosystems can thus be classified according to their degree of resistance to the anthropogenic inputs based on the results of the physico-chemical parameters and the species diversity as follows: southern coast $>$ Ghannouch area $>$ northern coast.
\end{abstract}

Keywords: Sfax, Ghannouch, $\mathrm{PO}_{4}^{3-}$, copepod, anthropogenic pressure, indicators

\section{INTRDDUCTION}

Marine coastal ecosystems include intertidal and nearshore systems that are influenced by atmospheric, terrestrial and autochthonous processes (Kennedy et al., 2002; Carr et al., 2003; Ruttenberg \& Granek, 2011; Bahloul et al., 2015). These ecosystems are generally sensitive to changes in upstream terrestrial systems and to direct inputs (Ruttenberg \& Granek, 2011). Thus, they are undergoing significant and growing anthropogenic threats (Cloern, 2001; Newton et al., 2003; Beaugrand et al., 2010; Brander, 2010; Burrows et al., 2011; Bahri-Trabelsi et al., 2013; Bahloul et al., 2015; Serranito et al., 2016).

Zooplankton plays a pivotal role in aquatic food webs by transferring carbon to higher trophic levels, consuming microorganisms (bacteria, protists) and serving as a prey for fish and invertebrates (De-Young et al., 2004; Sampey et al., 2007; Ziadi et al., 2015). Zooplankton communities are known to quickly respond to fluctuations in environmental

Corresponding author:

Z. Drira

Email: zaherdrira@yahoo.fr factors particularly in coastal areas where the combination of land and marine influences drives strong spatiotemporal variability (Siokou-Frangou, 1996). Zooplankton can thus be considered as useful indicators of ecosystem health status (Hays et al., 2005; Longhurst, 2007). The presence or absence of certain zooplankton species may indicate the relative influence of different water types on ecosystem structures and may serve as an early indication of a biological response to environmental and climatic changes (Hays et al., 2005; Ziadi et al., 2015). Zooplankton signatures may characterize specific hydrographic conditions in most of the world's ecosystems. Several studies have been undertaken in the Gulf of Gabes regarding the characterization of local zooplankton assemblages (Drira et al., 2010a, b, 2014; Ben Ltaief et al., 2015, 2017). In this area, the functioning of the coastal environments is highly complex due to the interaction of various factors, i.e. water movements, tide currents, anthropogenic inputs, marine traffic and fishing activities (Drira et al., 2008, 2016; Feki et al., 2013). The Gulf of Gabes is subject to a variety of human activities (Bejaoui et al., 2004; Gargouri et al., 2011, 2015). These activities include urban settlements, industrial areas and intense maritime traffic, resulting in the discharge of industrial and municipal effluents enriched in nutrients and pollutants which might negatively 
affect the water quality and the state of the ecosystem (Bejaoui et al., 2004; Gargouri-Ben Ayed et al., 2007; Gargouri et al., 2011, 2015; Aloulou et al., 2012; Ben Salem et al., 2015; Ben Salem \& Ayadi, 2016; Drira et al., 2016, 2017).

The main goal of this study was to improve knowledge about the spatial distribution of zooplankton abundance and composition in a large spectrum of three coastal areas in the Gulf of Gabes characterized by different degrees of pollution: (1) the northern coast of Sfax city which is an area restored via the Taparura project (Callaert et al., 2009); (2) the southern coast of Sfax city; and (3) Ghannouch coast, close to Gabes city, which is considered as highly polluted. The second aim was to relate the differences observed between the three sampled areas in respect of zooplankton abundance with physical (temperature, salinity and $\mathrm{pH}$ ), chemical (ammonium ions $\left(\mathrm{NH}_{4}^{+}\right)$, nitrates $\left(\mathrm{NO}_{3}^{-}\right)$, nitrites $\left(\mathrm{NO}_{2}^{-}\right)$, total nitrogen $(\mathrm{T}-\mathrm{N})$, orthophosphate $\left(\mathrm{PO}_{4}^{3-}\right)$, total phosphorus (T-P) and silicon atoms ions $\mathrm{Si}(\mathrm{OH})_{4}$ ) and biogeochemical (suspended particulate matter (SPM), particulate organic carbon and nitrogen (POC and PON), chlorophyll- $a$ (chl- $a$ ) and phaeopigment- $a$ (Phaeo- $a)$ ) water parameters characterizing the trophic and pollution status of each zone.

\section{MATERIALS AND METHODS}

\section{Study area}

The Gulf of Gabes (Eastern Mediterranean Sea, between $35^{\circ} \mathrm{N}$ and $33^{\circ} \mathrm{N}$, Tunisia), is endowed with rich aquatic resources contributing to about $65 \%$ of the national fish production in Tunisia (DGPA, 2004). Sampling was carried out in the Gulf of Gabes during October and November 2014, in three coastal areas i.e. the southern and the northern coasts of Sfax and the Ghannouch area. Thirty stations were sampled in the Gulf of Gabes among which 10 sampling stations were chosen for each area (SC, NC and GA) taking into account the pollution gradient (Figure 1).

The coastline of Sfax concentrates a great number of industrial activities, mainly related to phosphates, salt works, tanneries, lead foundry, textiles, ceramics industry, soap factories and building materials (Barhoumi et al., 2009).

The southern coast of Sfax (hereafter called SC) lies between the fishing harbour in the north and Gargour village in the south. This area is marked by the presence of the Société Industrielle d'Acide Phosphorique et d'Engrais

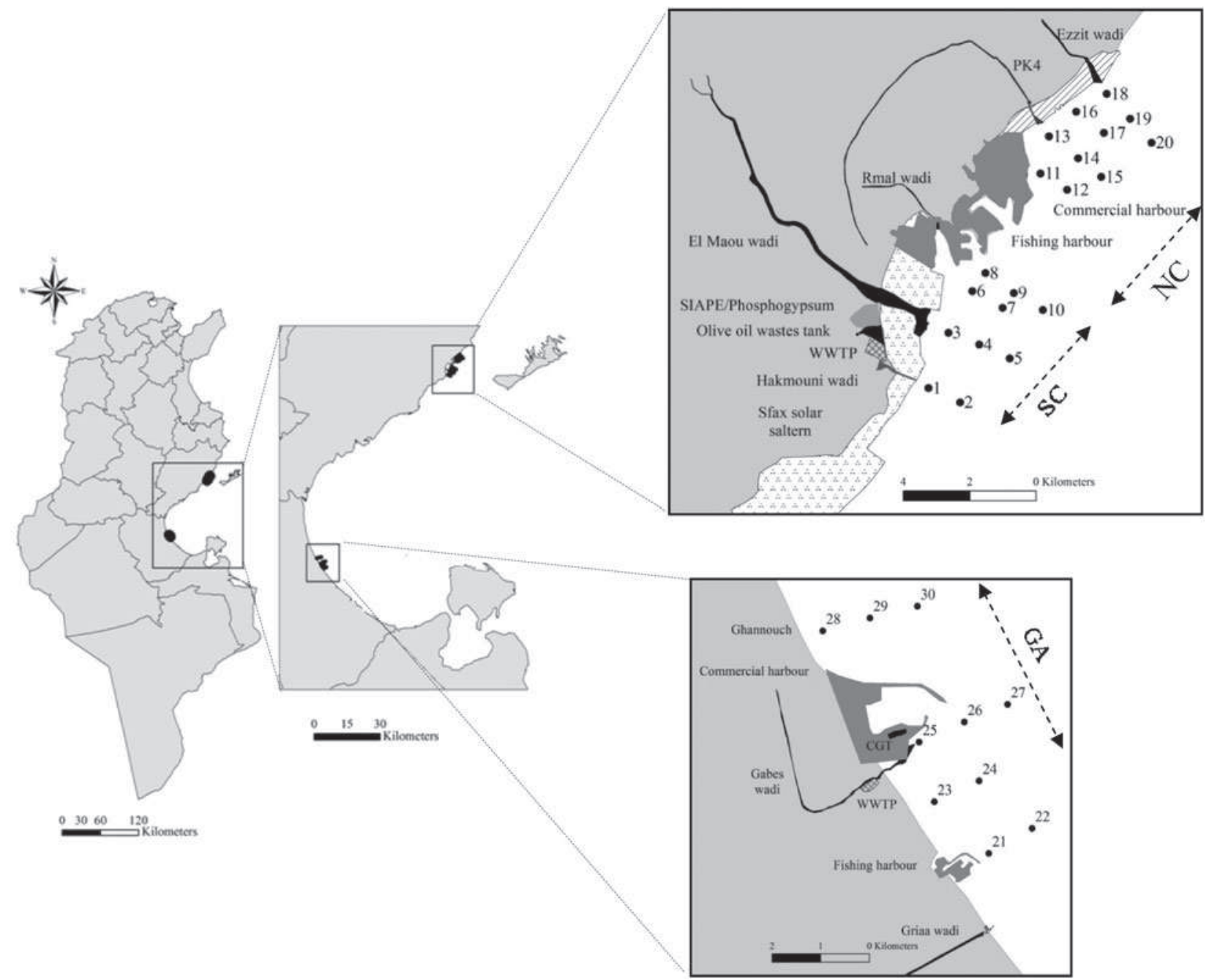

Fig. 1. Location of the studied stations in the southern (stations 1-10) and northern (stations 11-20) coastal areas of Sfax and the Ghannouch area (stations 21 -30) sampled during autumn (October-November 2014). 
(SIAPE) industry, which has released large amounts of phosphogypsum wastes for 40 years. These phosphogypsum wastes are a significant source of phosphates $\left(\mathrm{PO}_{4}^{3-}\right)$, chloride $\left(\mathrm{Cl}^{-}\right)$ and sulphates $\left(\mathrm{SO}_{4}^{2-}\right)$ for seawater and may explain the high chemical oxygen demand (COD) in the SC surface waters (Bahloul et al., 2015; Drira et al., 2016). Besides the SIAPE industry and its phosphogypsum wastes, the SC comprises several industrial areas related to textiles, tanneries, salt, olive oil, food processing, construction materials, ceramics and glass. Hence, several industrial effluents are released to the sea in this area. All these anthropogenic inputs have been shown to alter the marine environment and biodiversity in SC (Zaghden et al., 2005; Gargouri, 2006; Aloulou et al., 2012; Rekik et al., 2013; Bahloul et al., 2015).

The northern coast of Sfax (hereafter called NC), extending from the commercial harbour to wadi Ezzit and beyond, also suffers from the pressure of human activities (Hamza-Chaffai et al., 1997; Tayibi et al., 2009) and is subjected to increasing eutrophication with both red (Louati et al., 2001) and green tides caused by coastal Ulva rigida replacing the Posidonia oceanica seagrass beds (Ben Brahim et al., 2010). Previous studies in NC have focused on the sources and distribution of hydrocarbons in sediments (Louati et al., 2001; Zaghden et al., 2005) and marine bivalves (Hamza-Chaffai et al., 2003).

This area was recently restored through the Taparura project (2006-present), which aimed at remediating this part of Sfax city's coast. The project included the rehabilitation of a former complex industrial site, the reclamation of beaches and restoration of the area (Callaert et al., 2009). It led to significant improvement of plankton communities and water quality (Rekik et al., 2013, 2015). Indeed, this zone was strongly polluted by the phosphogypsum wastes from the NPK phosphoric acid industry, situated near the commercial harbour. The NPK was closed in 1992 and the Taparura project allowed the burial and confinement of the phosphogypsum wastes and the rehabilitation of the area between the commercial harbour and Sidi Mansour. In the NC, there are also the outlet of the rainwater drainage channel ('PK4'), which crosses the city from south-west to north-east, the outlet of the wadi Ezzit, which receives untreated domestic and industrial effluents.

Ghannouch area (hereafter called GA) includes a chemical industry complex as well as a commercial harbour, located $3 \mathrm{~km}$ north of Gabes city (Bejaoui et al., 2004). This complex houses the 'GCT-Gabes' phosphoric acid industry. Contrary to the SIAPE which stores its phosphogypsum wastes on land in an unprotected dome, the GCT-Gabes directly discharges its phosphogypsum wastes into the sea via an open channel. Organic pollution and drastic pollution by phosphate coming from the discharged sewage waters of the chemical plants of Ghannouch (Zaouali, 1993) favoured the emergence of green tides and Valonia to Ulva and the red tide or phytoplankton bloom (Hamza-Chaffai et al., 1995). This pollution also caused the disappearance of Caulerpa meadows, regression of Posidonia seagrass beds and decreasing diversity of the benthic fauna (Zaouali, 1993). Besides chemical industries, trawling practices (shrimps fishing) contribute to the deterioration of the Gabes ecosystem as well (Zaouali, 1993).

\section{Sampling and on board measurements}

Sampling was performed on board the vessel 'Taparura' between 10:30 am and 3:30 pm (18 and 23 October 2014;
SC and NC, respectively), 8:30 am and 12:30 am (13 November 2014; GA) around high tide and under conditions of calm sea and sunny weather. Seawater samples were collected at $\sim 0.1 \mathrm{~m}$ depth using $41 \mathrm{Nalgene}{ }^{\circledR}$ polycarbonate bottles. The bottles were opened below the water surface to avoid sampling of the surface microlayer. They were extensively washed with $1 \mathrm{M}$ hydrochloric acid $(\mathrm{HCl})$ and Milli-Q water before use, rinsed three times with the respective sample before filling and placed in the cold and in the dark after collection.

Zooplankton was collected using a cylindro-conical net ( $30 \mathrm{~cm}$ aperture, $100 \mathrm{~cm}$ height, $100 \mu \mathrm{m}$ mesh size) equipped with an Hydro-Bios flowmeter. The net was towed obliquely from near bottom to surface at each station at a mean speed of $1 \mathrm{~m} \mathrm{~s}^{-1}$ during 4 mins. After collection, zooplankton samples $(200 \mathrm{ml})$ were rapidly preserved in a buffered formaldehyde solution (2\%). They were stained with Rose Bengal to identify the internal tissues of the different zooplankton species and also to facilitate copepod dissection. In situ measurements of temperature, salinity and $\mathrm{pH}$ were carried out with measuring cells type TetraCon ${ }^{\circledR} 4$-electrode system and a refractometer.

\section{Filtration, chemical and biogeochemical analyses and zooplankton identification}

Back in the laboratory, samples were immediately filtered under a low vacuum $(<50 \mathrm{~mm} \mathrm{Hg})$ through pre-combusted $\left(500^{\circ} \mathrm{C}, 4 \mathrm{~h}\right) \mathrm{GF} / \mathrm{F}(\sim 0.7 \mu \mathrm{m})$ glass fibre filters $(25$ or $47 \mathrm{~mm}$ diameter, Whatman) using glassware filtration systems. Nutrients, i.e. $\mathrm{NO}_{2}^{-}, \mathrm{NO}_{3}^{-}, \mathrm{NH}_{4}^{+}, \mathrm{PO}_{4}^{3-}, \mathrm{Si}(\mathrm{OH})_{4}$, T-N and T-P, were analysed with a BRAN and LUEBBE type 3 autoanalyser and their concentrations were determined colorimetrically using a UV-visible 6400/6405 spectrophotometer according to the 'Standard Methods for the Examination of Water and Wastewater' (APHA, 1992).

For Chl- $a$ and Phaeo- $a$ analyses, 250-300 $\mathrm{ml}$ of samples were filtered. Filters were then extracted with methanol (RP prolabo) according to Raimbault et al. (2004). After $30 \mathrm{~min}$ of extraction in the dark at $4{ }^{\circ} \mathrm{C}$, a fluorescence measurement was performed with a fluorometer model 10 Turner Designs (Sunnyvale, USA) at $\lambda_{\mathrm{Ex}} / \lambda_{\mathrm{Em}}$ of $450 / 660 \mathrm{~nm}$. The acidification method was applied to determine Phaeo- $a$ concentrations. The fluorometer was calibrated with solutions of methanol (96\%) and Chl- $a$ (Sigma C5753). For SPM, POC and PON between 250 and $1100 \mathrm{ml}$ of sample were filtered with preweighted GF/F filters (the same filter was used for SPM, POC and PON analyses). After filtration, filters were dried at $60^{\circ} \mathrm{C}$ for $24 \mathrm{~h}$ and reweighed on the same balance. SPM concentration was calculated as the difference between filter weight before and after sample filtration, normalized to the filtration volume (Neukermans et al., 2012). POC and PON quantification were performed simultaneously with an autoanalyser II Technicon (New York, USA), using the wet-oxidation procedure according to Raimbault et al. (1999). POC and PON had a detection limit of 0.50 and $0.10 \mu \mathrm{m}$, respectively.

Zooplankton samples were identified according to Rose (1933), Bradford-Grieve (1999) and Costanzo et al. (2007). The different copepod species were sorted into four demographic classes (nauplii, copepodids, adult males and adult females). Miscellaneous zooplankton were also counted 
according to Tregouboff \& Rose (1978a, b). Enumeration was performed under a vertically mounted deep-focus dissecting microscope (Olympus TL 2) and numerical density was expressed in individual $\mathrm{m}^{-3}$. Total length of body size for the adult copepod was measured for each species in each sampled station (10 individuals for each species in each sampling set).

\section{Data processing and statistical analysis}

We applied the Geographic Information Systems (GIS) tools using ArcGIS 10.2 version software to make contour plots. Kriging was the method used to build maps relative to spatial distribution for all dataset parameters. Mesozooplankton diversity was measured using a range of univariate and multivariate diversity measurements. Species diversities were assessed using the Shannon diversity index $H^{\prime}$ (Shannon \& Weaver, 1949) and using the formula proposed by $J^{\prime}$ Pielou's evenness index (1966):

$$
\begin{gathered}
H^{\prime}=-\sum_{n i}^{i=1} \frac{n i}{N} \log _{2} \frac{n i}{N}, \\
J^{\prime}=H^{\prime} / \log _{2} S
\end{gathered}
$$

where $n_{i}$ is the number of individuals belonging to the species $i$ and $N$ is the total number of individuals in each station.

To identify the suitable environmental health indicator of these three coastal marine areas under contrasting anthropogenic inputs, we calculated the Indicator Value (IndVal) for each taxa as per Dufrene \& Legendre (1997) as used recently in Hemraj et al. (2017). Indicator species of each station was extracted by an indicator species analysis (Dufrene \& Legendre, 1997). The highest indicator value for given species was saved as a summary of the overall indicator value of that species. The IndVal of each species was computed as follows:

$$
\text { IndVal }=R A_{k j} \times R F_{k j} \times 100
$$

where $R A_{k j}$ is the relative abundance of species $j$ in group $k$, and $R F_{k j}$ is the relative frequency (presence/absence) of species $j$ in group $k$.

All statistical analyses were conducted using the XLStat 2014 software. ANOVA was applied to identify significant differences between these three sampled areas for physicochemical and biogeochemical variables. The spatial variability of copepod communities in relation to environmental variables was assessed using multivariate analysis after data transformation $\left[\log _{10}(x+1)\right]$ (Sokal \& Rohlf, 1981). Moreover, to explain the relationship between physico-chemical (depth, temperature, salinity and $\mathrm{pH})$, chemical $\left(\mathrm{NO}_{3}^{-}, \mathrm{NO}_{2}^{-}, \mathrm{NH}_{4}^{+}\right.$, $\mathrm{PO}_{4}^{3-}$, T-N, T-P, N/P ratio and $\left.\mathrm{Si}(\mathrm{OH})_{4}\right)$ and biogeochemical (copepods, chl- $a$ and SPM) parameters, we used a canonical correspondence analysis (CCA) (Ter-Braak, 1986) assessed by over 30 observations ( 30 stations). Pearson's rank correlations were used to determine the potential correlations between the copepod community and the physicobiogeochemical variables.

\section{RESULTS \\ Physico-chemical and biogeochemical parameters}

Mean values \pm standard deviation (SD) of physico-chemical and biogeochemical parameters recorded in surface waters of the three studied areas are given in Table 1 . Surface water temperature was warmer in SC $\left(26.8 \pm 0.23^{\circ} \mathrm{C}\right)$ than in $\mathrm{NC}\left(21.91 \pm 0.7^{\circ} \mathrm{C}\right)$ and GA $\left(19.8 \pm 1.68^{\circ} \mathrm{C}\right)$ (Table 1 ; Figure $2 \mathrm{~A}$ ) and the difference was significant (ANOVA, $P<0.0001)$. The highest temperature $\left(27.3^{\circ} \mathrm{C}\right)$ was recorded at station 10 from the SC and the lowest one $\left(18^{\circ} \mathrm{C}\right)$ at stations 21-24 from the GA (Table 1; Figure 2A). Salinity averaged at $38.4 \pm 3.4$ psu, varying from 32 psu at stations 3 (SC), 25 and 30 (GA) to $45 \mathrm{psu}$ at stations 16 (NC). It was significantly higher in the $\mathrm{NC}$ than in the two other areas (ANOVA, $P<0.0001$ ) (Table 1; Figure 2B). $\mathrm{pH}$ (mean value of $8.05 \pm 0.08)$ was higher in the $\mathrm{NC}(8.11 \pm 0.06)$ than in the GA $(8.04 \pm 0.07)$ and the SC $(7.99 \pm 0.07)$ (ANOVA, $P<0.0001$ ) (Table 1; Figure $2 \mathrm{C}$ ).

The concentration of total nitrogen $(\mathrm{T}-\mathrm{N})$ averaged $15.8 \pm$ $4 \mu \mathrm{m}$ and varied from 11.6 (station $25, \mathrm{GA}$ ) to $28.9 \mu \mathrm{m}$ (station 13, NC) with no significant difference between sites (ANOVA, $P>0.6$ ) (Table 1; Figure $3 \mathrm{~A}$ ). The relatively important T-N concentrations were due to the high contribution of $\mathrm{NH}_{4}^{+}$, close to $66 \%$ of T-N, which displayed a mean concentration of $5.2 \pm 1.6 \mu \mathrm{m}$ and showed highest value in the SC (ANOVA, $P<0.001$ ) (Table 1 ; Figure $3 \mathrm{~B}$ ). $\mathrm{NO}_{3}^{-}$concentration was also quite high, ranging from 1.3 (station $10 ; \mathrm{SC}$ ) to $11.4 \mu \mathrm{m}$ (station 13; NC), while $\mathrm{NO}_{2}^{-}$concentration was much lower (0.03-2.6 $\mu \mathrm{m}$, stations 25, GA and 13, NC) (Table 1; Figure $3 \mathrm{C}, \mathrm{D})$. Both oxidized nitrogen forms did not vary significantly between the three areas $(P>0.4)$. The concentration of total phosphorus (T-P) was on average $13.1 \pm 5.7 \mu \mathrm{m}$, ranging from 5 (station 2) to 25.1 (station 24) $\mu \mathrm{m}$ (Table 1 ; Figure $3 \mathrm{E})$ with highest values in $\mathrm{GA}(P=0.05)$. $\mathrm{PO}_{4}^{3-}$ concentration was on average $3.2 \pm 2.4 \mu \mathrm{m}$ with minimal and maximal values $0.5-9.6 \mu \mathrm{m}$ at stations 2 and 24 , respectively and no significant difference between sites $(P>0.05)$ (Table 1 ; Figure $3 \mathrm{~F}$ ). The $\mathrm{N} / \mathrm{P}$ ratio varied between 2.5 (station 7) and 30.3 (station 1) and was significantly higher in the SC than in the two other sites $(P<0.05)$ (Table 1 ; Figure $\left.{ }_{3} \mathrm{G}\right)$. $\mathrm{Si}(\mathrm{OH})_{4}$ concentration was on average $5.2 \pm$ $4.1 \mu \mathrm{m}$ with minimal and maximal values $1.5-19.5 \mu \mathrm{m}$ at stations 1 and 26, respectively $(P>0.9)$ (Table 1 ; Figure $3 \mathrm{H})$. SPM showed a mean value of $19.8 \pm 11 \mathrm{mg} \mathrm{l}^{-1}$ and varied between 8.5 and $59.5 \mathrm{mg} \mathrm{l}^{-1}$ at stations 5 and 16 , respectively with no significant difference between sites $(P>0.1)$ (Table 1$)$. Chl- $a$ concentration was higher in the SC $\left(11.7 \pm 13 \mu \mathrm{gl}^{-1}\right)$ than in the GA $\left(6.5 \pm 1.7 \mu \mathrm{gl}^{-1}\right)$ and the $\mathrm{NC}\left(5.1 \pm 4.2 \mu \mathrm{gl}^{-1}\right)$ (Table 1) but the differences were not significant $(P>0.1)$. Phaeo- $a$ concentration was higher in the SC $\left(3.3 \pm 3 \mu \mathrm{gl}^{-1}\right)$ than in the NC $\left(1.6 \pm 1 \mu \mathrm{gl}^{-1}\right)$ and the GA $(1.4 \pm$ $\left.0.4 \mu \mathrm{g} \mathrm{l}^{-1}\right)$ with no significant difference between zones $(P>$ 0.5) (Table 1). POC and PON showed a similar trend, with minimal and maximal concentrations in GA and NC, respectively; but with no significant difference between zones $(P>$ 0.2). $\mathrm{C} / \mathrm{N}$ ratio, averaged $5.9 \pm 0.9 \mu \mathrm{gl}^{-1}$, with higher mean values in GA $\left(6.4 \pm 0.5 \mu \mathrm{gl}^{-1}\right)$ than in $\mathrm{NC}\left(6.1 \pm 1 \mu \mathrm{gl}^{-1}\right)$ and in SC $\left(5.1 \pm 0.6 \mu \mathrm{gl}^{-1}\right)(P<0.001)$ (Table 1$)$. 
Table 1. Mean values and standard deviation (SD) of physico-chemical and biogeochemical parameters of 30 stations sampled in the northern and southern coastal areas of Sfax and the Ghannouch area sampled in October-November 2014. In the last column, results of ANOVA test for the comparison between these three sampled areas. Asterisks denote significant differences between different sampled areas: ${ }^{*} P<0.05 ;{ }^{* *} P<$ $0.001 ;{ }^{* * *} P<0.0001$.

\begin{tabular}{|c|c|c|c|c|c|c|c|c|c|c|c|c|}
\hline \multirow[t]{2}{*}{ Parameters } & \multicolumn{3}{|c|}{ Southern coastal area } & \multicolumn{3}{|c|}{ Northern coastal area } & \multicolumn{3}{|c|}{ Ghannouch area } & \multirow{2}{*}{$\begin{array}{l}\text { Stations with } \\
\text { min value }\end{array}$} & \multirow{2}{*}{$\begin{array}{l}\text { Stations with } \\
\text { max value }\end{array}$} & \multirow[t]{2}{*}{$F(P$ values $)$} \\
\hline & Min & $\operatorname{Max}$ & Mean \pm SD & Min & $\operatorname{Max}$ & Mean \pm SD & Min & Max & Mean \pm SD & & & \\
\hline \multicolumn{13}{|l|}{ Physical and chemical parameters } \\
\hline Depth $(\mathrm{m})$ & 1 & 9 & $4.9 \pm 2.7$ & 0.9 & 2 & $1.6 \pm 0.5$ & 2.8 & 10.5 & $8.0 \pm 2.4$ & 18 & 22 & $23.778(0.0001)^{* * *}$ \\
\hline Temperature $\left({ }^{\circ} \mathrm{C}\right)$ & 26.5 & 27.3 & $26.8 \pm 0.23$ & 21 & 23.2 & $21.91 \pm 0.7$ & 18 & 22 & $19.8 \pm 1.68$ & $21-24$ & 10 & $8.617(0.001)^{* * *}$ \\
\hline Salinity (psu) & 32 & 40 & $38.0 \pm 2.7$ & 38 & 45 & $41.5 \pm 2.07$ & 32 & 40 & $35.7 \pm 2.7$ & $3,25,30$ & 16 & $12,781(0.0001)^{* * *}$ \\
\hline $\mathrm{Ph}$ & 7.9 & 8.11 & $7.99 \pm 0.07$ & 8 & 8.21 & $8.11 \pm 0.06$ & 7.97 & 8.14 & $8.04 \pm 0.07$ & 1 & 17 & $8.645(0.001)^{* * *}$ \\
\hline \multicolumn{13}{|l|}{ Biogeochemical parameters } \\
\hline $\mathrm{NO}_{3}^{-}(\mu \mathrm{m})$ & 1.31 & 7.51 & $3.15 \pm 1.92$ & 1.71 & 11.44 & $3.07 \pm 2.95$ & 1.33 & 3.1 & $2.13 \pm 0.48$ & 10 & 13 & $0.905(0.417)$ \\
\hline $\mathrm{NO}_{2}^{-}(\mu \mathrm{m})$ & 0.03 & 0.88 & $0.25 \pm 0.3$ & 0.04 & 2.64 & $0.37 \pm 0.8$ & 0.04 & 0.35 & $0.15 \pm 0.1$ & 25 & 13 & $0.315(0.733)$ \\
\hline $\mathrm{NH}_{4}^{+}(\mu \mathrm{m})$ & 5.48 & 10.22 & $6.42 \pm 1.36$ & 2.99 & 7.57 & $5.01 \pm 1.71$ & 3.27 & 5.21 & $4.14 \pm 0.7$ & 19 & 1 & $8.297(0.002)^{* * *}$ \\
\hline $\mathrm{T}-\mathrm{N}(\mu \mathrm{m})$ & 12.98 & 26.31 & $16.41 \pm 3.96$ & 11.99 & 28.98 & $16.16 \pm 5.4$ & 11.61 & 18.28 & $14.72 \pm 2.35$ & 25 & 13 & $0.472(0.629)$ \\
\hline $\mathrm{PO}_{4}^{3-}(\mu \mathrm{m})$ & 0.45 & 7.92 & $3.11 \pm 2.82$ & 1.25 & 2.97 & $2.07 \pm 0.62$ & 1.3 & 9.56 & $4.46 \pm 2.6$ & 2 & 24 & $2.661(0.088)$ \\
\hline $\mathrm{T}-\mathrm{P}(\mu \mathrm{m})$ & 4.95 & 24.14 & $13.49 \pm 7.25$ & 8.02 & 11.26 & $9.75 \pm 1.26$ & 9.95 & 25.13 & $16 \pm 5.42$ & 2 & 24 & $3.347(0.050)^{*}$ \\
\hline $\mathrm{Si}(\mathrm{OH})_{4}(\mu \mathrm{m})$ & 1.56 & 9.41 & $4.38 \pm 3.2$ & 3.58 & 17.48 & $5.92 \pm 4.18$ & 2.48 & 19.48 & $5.37 \pm 5$ & 1 & 26 & $0.926(0.408)$ \\
\hline $\mathrm{N} / \mathrm{P}$ ratio & 2.50 & 30.29 & $9.57 \pm 8.50$ & 3.30 & 16.74 & $6.07 \pm 3.95$ & 2.57 & 7.45 & $3.66 \pm 1.50$ & 7 & 1 & $3.889(0.033)^{*}$ \\
\hline $\operatorname{SPM}\left(\mathrm{mg} \mathrm{l}^{-1}\right)$ & 8.31 & 32.17 & $15.4 \pm 8.42$ & 9.66 & 59.38 & $24.14 \pm 15.56$ & 16.98 & 22.66 & $20.12 \pm 1.87$ & 5 & 16 & $1.970(0.158)$ \\
\hline Chlorophyll- $a\left(\mu \mathrm{g} \mathrm{l}^{-1}\right)$ & 2 & 40.7 & $11.7 \pm 13$ & 1.7 & 16.1 & $5.1 \pm 4.2$ & 2.95 & 8.7 & $6.5 \pm 1.7$ & 14 & 8 & $1.935(0.164)$ \\
\hline Phaeopigment- $a\left(\mu \mathrm{g}^{-1}\right)$ & 0.43 & 9.70 & $3.32 \pm 3.07$ & 0.57 & 3.53 & $1.62 \pm 1.02$ & 0.65 & 2.09 & $1.44 \pm 0.40$ & 10 & 8 & $3.043(0.064)$ \\
\hline $\operatorname{POC}\left(\mu \mathrm{g} \mathrm{l}^{-1}\right)$ & 178.43 & 2129.44 & $764.73 \pm 722.88$ & 283.49 & 2937.04 & $1013.80 \pm 942.12$ & 294.37 & 657.88 & $487.84 \pm 113.94$ & 10 & 18 & $1.459(0.25)$ \\
\hline $\operatorname{PON}\left(\mu \mathrm{gl}^{-1}\right)$ & 31.18 & 483.85 & $160.32 \pm 167.73$ & 58.19 & 522.32 & $163.22 \pm 152.86$ & 41.61 & 107.48 & $76.76 \pm 18.39$ & 10 & 18 & $1.395(0.265)$ \\
\hline $\mathrm{C} / \mathrm{N}$ ratio & 4.40 & 6.32 & $5.17 \pm 0.60$ & 4.70 & 7.69 & $6.08 \pm 1.08$ & 5.85 & 7.07 & $6.39 \pm 0.46$ & 6 & 13 & $6.840(0.004)^{* *}$ \\
\hline \multicolumn{13}{|l|}{ Zooplankton } \\
\hline $\begin{array}{l}\text { Total zooplankton } \\
\left(\times 10^{3} \text { ind } \mathrm{m}^{-3}\right)\end{array}$ & 6 & 157.32 & $61.73 \pm 51.73$ & 2.57 & 20.85 & $7.73 \pm 5.5$ & 4.82 & 28.36 & $15.77 \pm 6.64$ & 16 & 4 & $11.170(0.000)^{* * *}$ \\
\hline $\begin{array}{l}\text { Non-copepod zooplankton } \\
\left(\times 10^{3} \text { ind } \mathrm{m}^{-3}\right)\end{array}$ & 0.8 & 29.5 & $10.35 \pm 9.04$ & 0.18 & 4.1 & $1.21 \pm 1.28$ & 1.11 & 7.2 & $3.79 \pm 1.79$ & 16 & 4 & $12.604(0.000)^{* * *}$ \\
\hline Total copepods $\left(\times 10^{3}\right.$ ind $\left.\mathrm{m}^{-3}\right)$ & 5.2 & 127.82 & $51.37 \pm 43.41$ & 2.39 & 16.73 & $6.51 \pm 4.3$ & 3.33 & 24.18 & $11.98 \pm 5.65$ & 16 & 4 & $10.675(0.000)^{* * *}$ \\
\hline Calanoids $\left(\times 10^{3}\right.$ ind $\left.\mathrm{m}^{-3}\right)$ & 0.41 & 21.9 & $9.13 \pm 8.5$ & 1.27 & 4.48 & $2.07 \pm 0.94$ & 1.28 & 13.73 & $5.8 \pm 3.65$ & 7 & 2 & $2.421(0.108)$ \\
\hline Harpacticoids $\left(\times 10^{3}\right.$ ind $\left.\mathrm{m}^{-3}\right)$ & o & 1.4 & $0.45 \pm 0.52$ & o & 0.65 & $0.25 \pm 0.25$ & 0.14 & 1.81 & $0.72 \pm 0.6$ & $8,9,13$ & 22 & $2.047(0.149)$ \\
\hline Cyclopoids $\left(\times 10^{3}\right.$ ind $\left.\mathrm{m}^{-3}\right)$ & 3.14 & 107.75 & $37.41 \pm 35.85$ & 0.78 & 5.08 & $2.54 \pm 1.49$ & 1.72 & 10 & $4.92 \pm 2.49$ & 16 & 4 & $17.203(0.0001)^{* * *}$ \\
\hline Adult male $\left(\times 10^{3}\right.$ ind $\left.\mathrm{m}^{-3}\right)$ & 0.69 & 27.983 & $6.98 \pm 8.44$ & 0.5 & 1.88 & $1.18 \pm 0.5$ & 0.13 & 5.19 & $1.18 \pm 1.48$ & 24 & 4 & $9.994(0.001)^{* * *}$ \\
\hline Adult female $\left(\times 10^{3}\right.$ ind $\left.\mathrm{m}^{-3}\right)$ & 0.95 & 60.05 & $22.27 \pm 19.97$ & 0.49 & 4.24 & $1.75 \pm 1.09$ & 0.6 & 8.63 & $6.07 \pm 2.84$ & 16 & 4 & $9.343(0.001)^{* * *}$ \\
\hline Copepodit $\left(\times 10^{3}\right.$ ind $\left.\mathrm{m}^{-3}\right)$ & 1.04 & 30.24 & $13.32 \pm 11.22$ & 0 & 0.003 & $0.001 \pm 0$ & 0.85 & 3.58 & $2.41 \pm 1.01$ & 16 & 4 & $257.789(0.0001)^{* * *}$ \\
\hline Nauplii $\left(\times 10^{3}\right.$ ind $\left.\mathrm{m}^{-3}\right)$ & 0.45 & 8.7 & $4.36 \pm 3.03$ & o & 0.006 & $0.001 \pm 0.002$ & 0 & 1.14 & $0.52 \pm 0.43$ & $\begin{array}{l}16,19-20,27, \\
30\end{array}$ & 4 & $45.899(0.0001)^{* * *}$ \\
\hline Length of copepod species (mm) & 0.70 & 0.72 & $0.71 \pm 0.004$ & 0.68 & 0.69 & $0.68 \pm 0.001$ & 0.82 & 0.83 & $0.82 \pm 0.001$ & 15 & 21 & $6816.340(0.000)^{* * *}$ \\
\hline Sex ratio & 0.13 & 2.35 & $0.63 \pm 0.81$ & 0.36 & 2.45 & $0.86 \pm 0.6$ & 0.01 & 0.83 & $0.27 \pm 0.28$ & 24 & 16 & $3.646(0.040)^{*}$ \\
\hline Number of copepod species & 9 & 18 & $12.6 \pm 2.59$ & 8 & 15 & $11.9 \pm 2.33$ & 5 & 12 & $8.1 \pm 2.18$ & 30 & 3 & $11.775(0.000)^{* * *}$ \\
\hline Shannon index (bits ind ${ }^{-1}$ ) & 0.96 & 1.94 & $1.49 \pm 0.33$ & 1.63 & 2.4 & $1.95 \pm 0.26$ & 1.24 & 2.21 & $1.74 \pm 0.28$ & 9 & 19 & $6.160(0.006)^{* *}$ \\
\hline
\end{tabular}



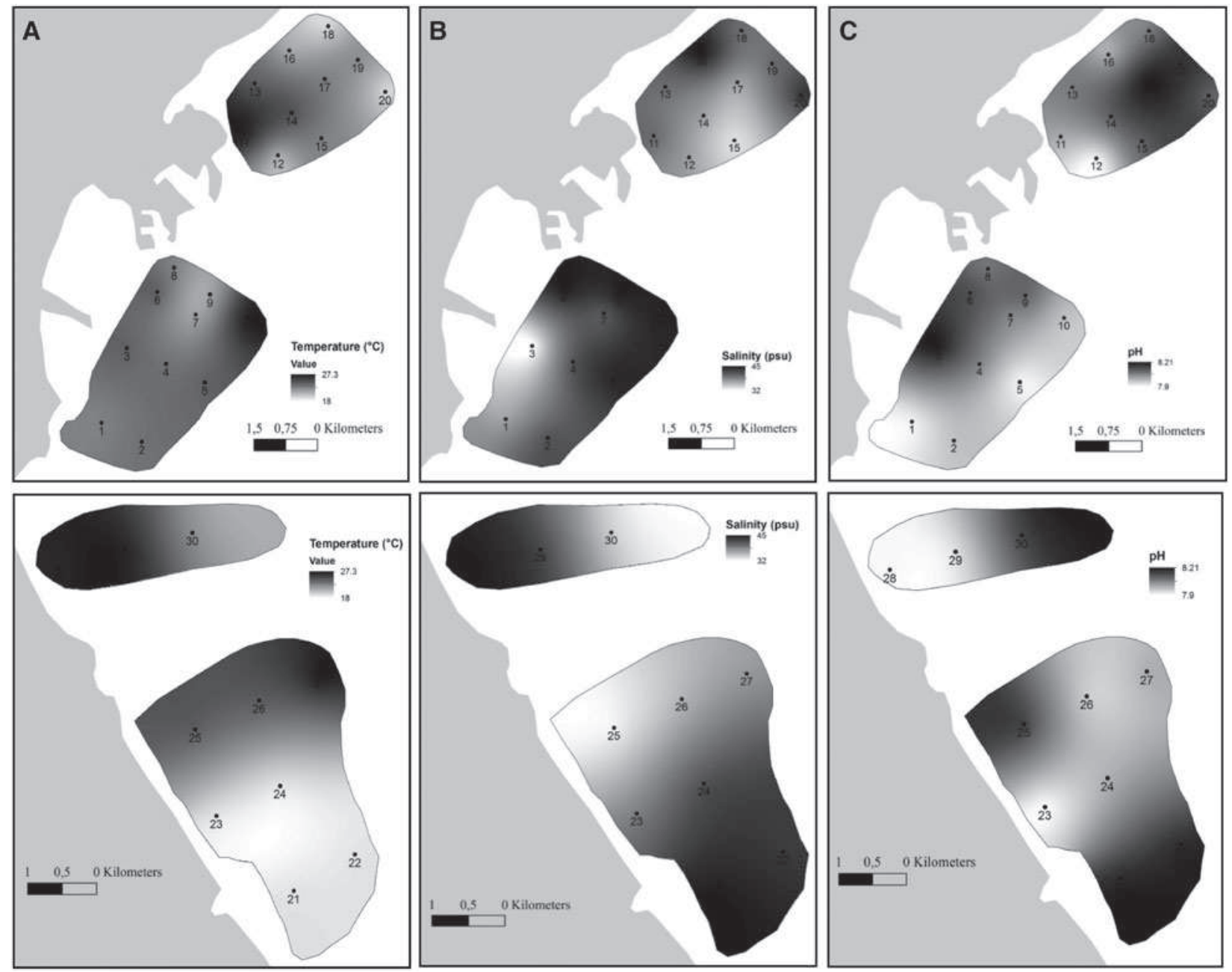

Fig. 2. Spatial variation of physical parameters, i.e. temperature (A), salinity (B) and $\mathrm{pH}(\mathrm{C})$ in stations sampled in the northern and southern coastal areas of Sfax and the Ghannouch area during autumn (October-November 2014).

\section{Zooplankton}

The total zooplankton abundance varied from $2.57 \times 10^{3}$ (station 16) to $157.32 \times 10^{3}$ ind $\mathrm{m}^{-3}$ (station 4) (Table 1 ; Figure 4A). Zooplankton assemblages were dominated by copepods which represented 82,80 and $75 \%$ of total zooplankton abundance, in SC, NC and GA, respectively (Table 2). The density of non-copepod zooplankton varied from $0.18 \times 10^{3}$ (station 16 ) to $29.5 \times 10^{3}$ ind $\mathrm{m}^{-3}$ (station 4) (Table 1; Figure $4 \mathrm{~B}$ ). Polychaete larvae, cirriped larvae, ostracods, jellyfish, zoea, fish eggs and gastropods were permanent components of meroplankton contributing to 90,67 and $85 \%$ of the non-copepod abundance, in SC, NC and GA, respectively (Table 2). On the other hand, appendicularians, cladocerans, foraminifera and amphipods were also permanent components of the holoplankton but did not exceed $33 \%$ of noncopepod abundance (Table 2). Total copepods varied from 2.39 (station 16) to $127.82 \times 10^{3}$ ind $\mathrm{m}^{-3}$ (station 4 ) (Table 1 ; Figure $4 \mathrm{C}$ ).

A total of 25 different copepod species were identified throughout the study period belonging to three different orders: Calanoida, Cyclopoida and Harpacticoida (Table 2; Figure $5 \mathrm{D}-\mathrm{F}$ ). Calanoida was the most diverse order (12 species) followed by Cyclopoida (seven species) and
Harpacticoida (six species), which contributed to 19,80 and $1 \%$, respectively to the total zooplankton abundance in SC, 43, 52 and 5\% in NC and 51, 43 and $6 \%$ in GA (Table 2). Among calanoid copepods, Paracalanus parvus (Claus, 1863) and Paracartia grani (Sars, 1904) were the most abundant species in SC (6 and 3.5\%) and NC (10 and $16 \%$ total zooplankton abundance), whereas Paracalanus parvus (22.5\% total zooplankton abundance) prevailed in GA. Among cyclopoid copepods, Oithona nana (Giesbrecht, 1892) and Oithona similis (Claus, 1866) were the most abundant species representing 59, 33 and $24 \%$ of total zooplankton abundance, in SC, NC and GA, respectively. Oithona similis was the only ubiquist and cosmopolitan species in this study period (100\% occurrence frequency) (Table 2). Conversely, Oithona setigera (Crisafi, 1959), Acamthocyclops sp. (Kiefer, 1927 ) and Paracartia latisetosa (Kritchagin, 1873) were specific to SC and totally absent in the two other areas, whereas Tisbe battagliai (Volkmann-Rocco, 1972) and Tigriopus sp. (Mori, 1932) were recorded only in NC. The main differences in copepod between the two sampled areas nearest in time (NC and SC) were found to be influenced by the water column depth. In fact, the noticeable presence of meiobenthic copepods such as Tigriopus and Tisbe were observed only in the shallower area (NC mean depth: $1.6 \mathrm{~m}$ ). However, the 

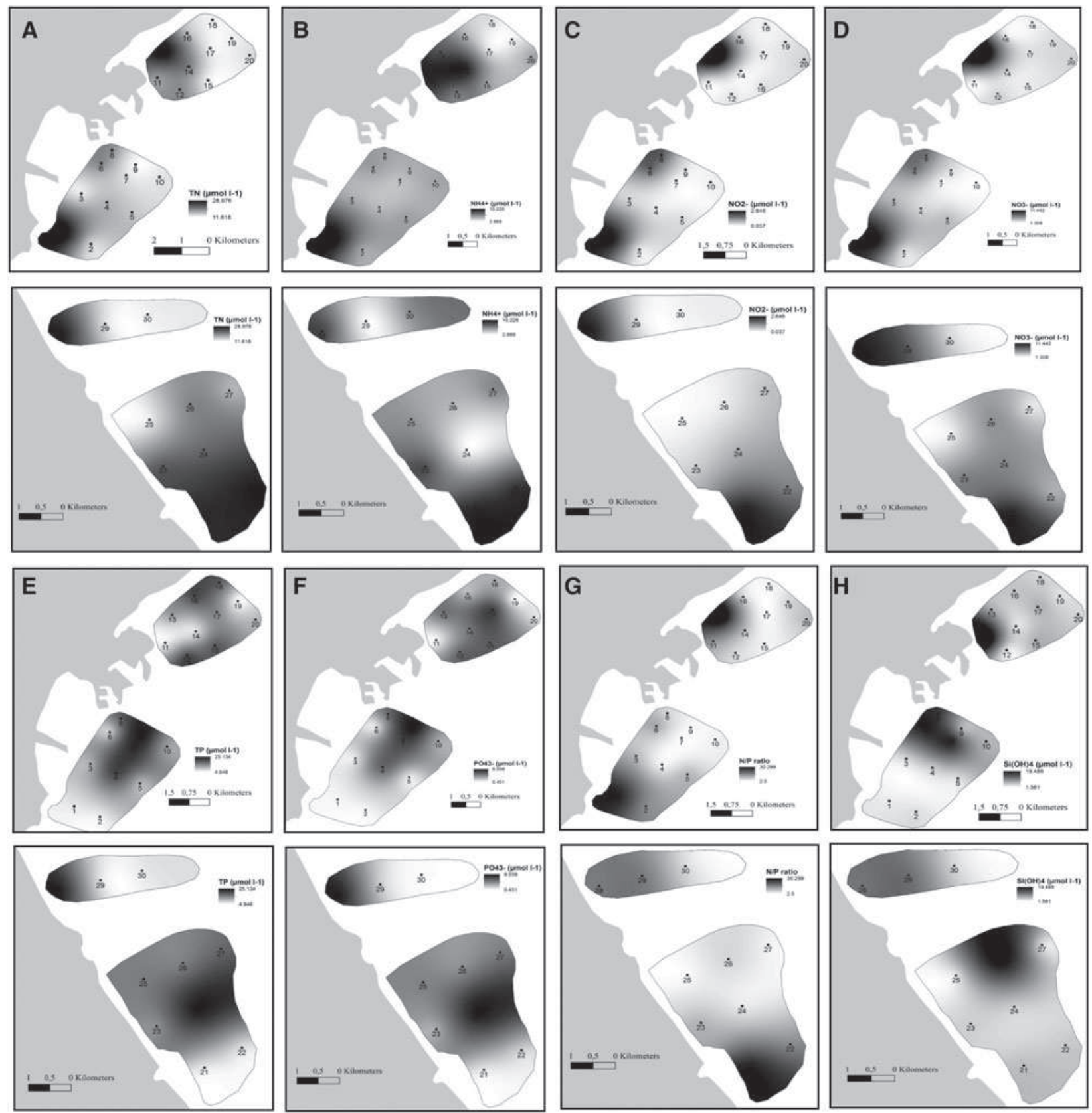

Fig. 3. Spatial variation of nutrient compounds, i.e. total nitrogen (T-N) $(\mathrm{A})$, ammonium $\left(\mathrm{NH}_{4}^{+}\right)(\mathrm{B})$, nitrite $\left(\mathrm{NO}_{2}^{-}\right)(\mathrm{C})$, nitrate $\left(\mathrm{NO}_{3}^{-}\right)(\mathrm{D})$, total phosphate $(\mathrm{T}-\mathrm{P})$ $(\mathrm{E})$, orthophosphate $\left(\mathrm{PO}_{4}^{3-}\right)(\mathrm{F}), \mathrm{N} / \mathrm{P}$ ratio $(\mathrm{G})$ and silicate $\left(\mathrm{Si}(\mathrm{OH})_{4}\right)(\mathrm{H})$ in stations sampled in the northern and southern coastal areas of Sfax and the Ghannouch area during autumn (October-November 2014).

highest abundance of all main copepod taxa was recorded in the deeper area (SC mean depth: $4.9 \mathrm{~m}$ ) which corroborates this fact (Tables $1 \& 2$, and CCA). All these species did not exceed $40 \%$ of occurrence frequency. Harpacticoids did not exceed $6 \%$ of total zooplankton abundance during the survey period and the highest abundance was observed with Euterpina acutifrons (Dana, 1847) in GA which represented $4.7 \%$ of total zooplankton (Table 2 ).

The abundance peak of copepods recorded at station 4 was associated with a high density of cyclopoids, adult male and female, copepodit and nauplii (Tables 1 \& 2; Figures $4 \mathrm{C} \&$ $\left.{ }_{5} \mathrm{C}-\mathrm{E}, \mathrm{G}-\mathrm{H}\right)$. During this period, low percentages of larval stages (copepodids: 29, 1 and 25\%, nauplii: 9, 22 and $6 \%$ of total copepod abundance) and high numbers of adults (62,
47 and $69 \%$ of total copepod abundance) were recorded at SC, NC and GA, respectively. The sex-ratio (adult male/ adult female) did not exceed 0.89 in most stations and was $>_{1}$ (male dominance) only at stations $3,7,16$ and 20 where it varied from 1.06 to 2.45 (Table 1 ; Figure $5 \mathrm{~F}$ ). ShannonWeaver diversity index $\left(\mathrm{H}^{\prime}\right)$ for copepods was relatively low with values ranging between 0.96 (10 species, station 9 in SC) and 2.4 bits ind $^{-1}$ (15 species, station 19 in NC) (Table 1; Figure $5 \mathrm{~A}$ ). Evenness index $(J)$ was higher in the GA $(0.6 \pm 0.1)$ and in NC $(0.6 \pm 0.08)$ than in SC $(0.5 \pm$ 0.1) (Figure $5 \mathrm{~B}$ ).

Most of the zooplankton parameters displayed significant differences between NC, SC and GA, except for calanoid and harpacticoid (ANOVA, $P<0.05$ ). Total zooplankton, 
Table 2. Quantitative aspects (D, Density; RA, Relative abundance; FO, Frequency of occurrence; TL, Total length) of the zooplankton taxa sampled from 30 stations of the northern and southern coastal areas of Sfax and the Ghannouch area sampled in October-November 2014 (Abbr: Abbreviation, ${ }^{*}$ : Large copepods with TL $>1.45 \mathrm{~mm}$ ).

\begin{tabular}{|c|c|c|c|c|c|c|c|c|c|c|c|c|c|}
\hline \multirow[t]{2}{*}{ Zooplankton } & \multirow[t]{2}{*}{ Abbr } & \multicolumn{4}{|c|}{ Southern coastal area } & \multicolumn{4}{|c|}{ Northern coastal area } & \multicolumn{4}{|c|}{ Ghannouch area } \\
\hline & & $D\left(\right.$ ind $\left.m^{-3}\right)$ & RA (\%) & $\begin{array}{l}\text { FO } \\
(\%)\end{array}$ & $\begin{array}{c}\text { TL } \\
(\mathbf{m m})\end{array}$ & $D\left(\right.$ ind $\mathrm{m}^{-3}$ ) & $\begin{array}{l}\text { RA } \\
(\%)\end{array}$ & $\begin{array}{l}\text { FO } \\
(\%)\end{array}$ & $\begin{array}{c}\mathrm{TL} \\
(\mathrm{mm})\end{array}$ & $D\left(\right.$ ind $\left.m^{-3}\right)$ & $\begin{array}{l}\text { RA } \\
(\%)\end{array}$ & $\begin{array}{l}\text { FO } \\
(\%)\end{array}$ & $\begin{array}{c}\mathrm{TL} \\
(\mathrm{mm})\end{array}$ \\
\hline Copepods & & & 82 & & & & 80 & & & & 75 & & \\
\hline Calanoids & & & 19 & & & & 43 & & & & 51 & & \\
\hline Acartia clausi (Giesbrecht, 1889) & Acl & $347.992 \pm 661.81$ & 0.61 & 80 & 1.02 & $19.626 \pm 31.962$ & 0.32 & 40 & 1.06 & - & - & - & - \\
\hline $\begin{array}{l}\text { Acartia discaudata (Giesbrecht, } \\
\text { 1882) }\end{array}$ & Adi & $1.2717 \pm 4.021$ & 0.002 & 10 & 1.20 & - & - & - & - & $15.543 \pm 49.151$ & 0.10 & 10 & 1.30 \\
\hline Acartia sp. (Dana, 1846) & Aca & $131.585 \pm 188.125$ & 0.23 & 70 & 1.14 & $20.799 \pm 42.937$ & 0.34 & 30 & 1.15 & $63.302 \pm 151.765$ & 0.42 & 20 & 1.16 \\
\hline Paracalanus parvus (Claus, 1863) & $P p a$ & $3296.7355 \pm 3667.654$ & 5.75 & 90 & 0.81 & $609.529 \pm 550.77$ & 10 & 100 & 0.84 & $3403.281 \pm 1743.181$ & 22.31 & 100 & 0.81 \\
\hline Paracartia grani (Sars, 1904) & $P g r$ & $1938.0378 \pm 2850.466$ & 3.38 & 90 & 1.13 & $953.156 \pm 245.02$ & 15.64 & 100 & 1.15 & $485.082 \pm 592.992$ & 3.18 & 60 & 1.14 \\
\hline $\begin{array}{l}\text { Paracartia latisetosa (Kritchagin, } \\
\text { 1873) }\end{array}$ & Pla & $1.526 \pm 4.825$ & 0.003 & 10 & 1.13 & - & - & - & - & - & - & - & - \\
\hline Temora longicornis (Müller, 1792) & Tlo & $125.851 \pm 356.164$ & 0.22 & 40 & 0.86 & $76.277 \pm 96.902$ & 1.25 & 60 & 0.88 & $510.234 \pm 849.184$ & 3.35 & 40 & 0.82 \\
\hline Temora stylifera (Dana, 1849) & Tst & - & - & - & - & $20.135 \pm 43.807$ & 0.33 & 20 & 0.85 & $641.855 \pm 1081.192$ & 4.21 & 60 & 0.85 \\
\hline Temora sp. (Baird, 1850) & Tsp & $346.019 \pm 494.655$ & 0.60 & 90 & 0.80 & - & - & - & - & $141.3 \pm 446.829$ & 0.93 & 10 & 0.82 \\
\hline $\begin{array}{l}\text { Centropages kröyeri (Giesbrecht, } \\
\text { 1893) }\end{array}$ & $C k r$ & $2010.816 \pm 2756.877$ & 3.51 & 90 & 0.78 & $277.133 \pm 283.274$ & 4.55 & 80 & 0.78 & $226.503 \pm 573.476$ & 1.49 & 30 & 0.76 \\
\hline $\begin{array}{l}\text { Aglaodiaptomus leptopus (Forbes, } \\
\text { 1882) }\end{array}$ & Ale & $0.1 \pm 0.316$ & 0.0002 & 10 & 0.86 & $8.901 \pm 19.337$ & 0.15 & 20 & $1.63^{*}$ & $269.883 \pm 411.052$ & 1.77 & 40 & $1.60^{*}$ \\
\hline Eucalanus sp. (Dana, 1852) & Esp & $933.814 \pm 1657.699$ & 1.63 & 100 & 0.69 & $90.752 \pm 85.856$ & 1.49 & 100 & 0.71 & $49.666 \pm 157.06$ & 0.33 & 10 & 0.68 \\
\hline Harpacticoids & & & 1 & & & & 5 & & & & 6 & & \\
\hline $\begin{array}{l}\text { Clytemnestra scutellata (Dana, } \\
1847 \text { ) }\end{array}$ & Csc & $45.165 \pm 110.298$ & 0.08 & 40 & 0.16 & $30.697 \pm 42.916$ & 0.50 & 70 & 0.18 & - & - & - & - \\
\hline Euterpina acutifrons (Dana, 1847) & Eac & $349.566 \pm 490.107$ & 0.61 & 80 & 0.25 & $52.832 \pm 69.231$ & 0.87 & 60 & 0.29 & $724.812 \pm 601.595$ & 4.75 & 100 & 0.27 \\
\hline Harpacticus littoralis (Sars, 1910) & $H l i$ & $52.945 \pm 70.244$ & 0.09 & 50 & 0.33 & $97.97 \pm 137.835$ & 1.61 & 60 & 0.35 & - & - & - & - \\
\hline $\begin{array}{l}\text { Tisbe battagliai (Volkmann-Rocco, } \\
\text { 1972) }\end{array}$ & $T b a$ & - & - & - & - & $59.035 \pm 94.881$ & 0.97 & 40 & 0.23 & - & - & - & - \\
\hline $\begin{array}{l}\text { Microsettela norvegica (Boeck, } \\
\text { 1865) }\end{array}$ & Mno & $10.061 \pm 27.981$ & 0.02 & 20 & 0.21 & $6.994 \pm 22.118$ & 0.11 & 10 & 0.26 & - & - & - & - \\
\hline Tigriopus sp. (Mori, 1932) & Tsp & - & - & - & - & $4.38 \pm 9.253$ & 0.07 & 20 & 0.23 & - & - & - & - \\
\hline Cyclopoids & & & 80 & & & & 52 & & & & 43 & & \\
\hline Oithona nana (Giesbrecht, 1892) & Ona & $20,593.312 \pm 21,463.701$ & 35.90 & 100 & 0.57 & $777.948 \pm 597.39$ & 12.77 & 80 & 0.61 & $974.489 \pm 644.897$ & 6.39 & 100 & 0.56 \\
\hline Oithona plumifera (Baird, 1843) & Opl & $2829.25 \pm 4368.842$ & 4.93 & 100 & 0.63 & $367.42 \pm 224.239$ & 6.03 & 100 & 0.66 & $831.479 \pm 880.519$ & 5.45 & 80 & 0.63 \\
\hline Oithona similis (Claus, 1866) & Osi & $13,191.252 \pm 13,569.645$ & 22.99 & 100 & 0.48 & $1309.853 \pm 1014.211$ & 20.86 & 100 & 0.57 & $3120.144 \pm 1791.003$ & 17.27 & 100 & 0.52 \\
\hline Oithona sp. (Baird, 1843) & Osp & $637.283 \pm 1310.432$ & 1.11 & 40 & 0.48 & $24.031 \pm 49.854$ & 0.39 & 30 & 0.49 & - & - & - & - \\
\hline Oithona setigera (Crisafi, 1959) & Ose & $21.035 \pm 66.519$ & 0.04 & 10 & 0.51 & - & - & - & - & - & - & - & - \\
\hline $\begin{array}{l}\text { Acanthocyclops vernalis (Fischer, } \\
\text { 1853) }\end{array}$ & Ave & $144.291 \pm 442.027$ & 0.25 & 30 & 0.90 & $66.538 \pm 76.68$ & 1.09 & 50 & 1.01 & - & - & - & - \\
\hline Acanthocyclops sp. (Kiefer, 1927) & Asp & $2.289 \pm 7.238$ & 0.004 & 10 & 0.68 & - & - & - & - & - & - & - & - \\
\hline Non-copepod zooplankton & & & 18 & & & & 20 & & & & 25 & & \\
\hline Meroplankton & & & 90 & & & & 67 & & & & 85 & & \\
\hline Polychaeta larvae & Pla & $585.594 \pm 771.279$ & 1.0 & 100 & - & $82.276 \pm 75.335$ & 1.4 & 90 & - & $259.073 \pm 182.159$ & 1.7 & 80 & - \\
\hline Cirripedia larvae & Cla & $1227.195 \pm 1283.605$ & 2.1 & 100 & - & $245.18 \pm 321.581$ & 4.0 & 90 & - & $420.155 \pm 632.759$ & 2.8 & 60 & - \\
\hline
\end{tabular}




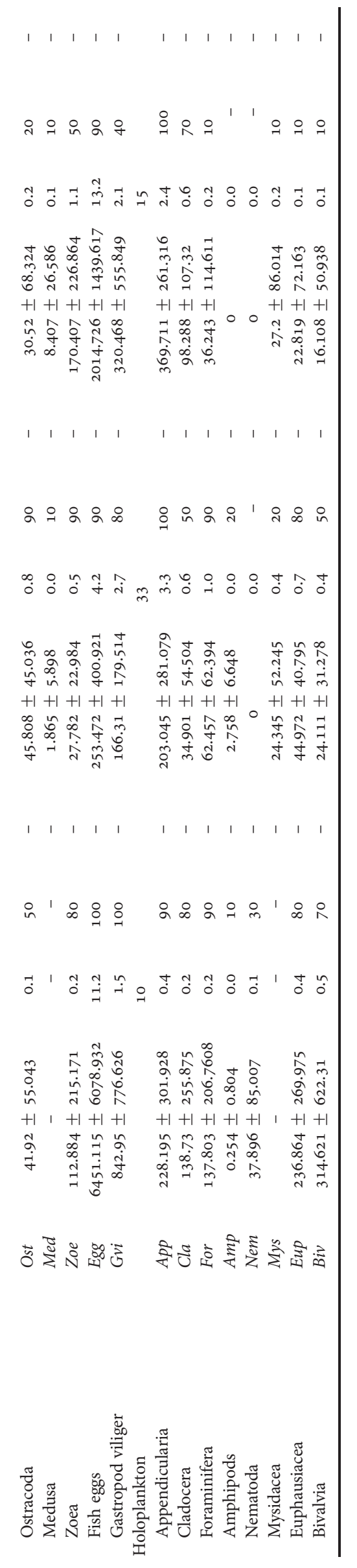

non-copepod zooplankton, total copepods, cyclopoid, adult males, adult females, copepodit, nauplii and number of copepod species were more abundant in SC than in NC and GA (ANOVA, $P<0.0001$ ) (Table 1). The lowest abundances were always recorded at NC with total zooplankton being 8 -fold and 2-fold less abundant than at SC and GA respectively and particularly low values for nauplii and copopodites almost absent in this area. The Shannon and Weaver diversity index $\left(\mathrm{H}^{\prime}\right)$ for copepods was significantly higher at $\mathrm{NC}$ than at $\mathrm{SC}$ and GA. The length of copepod species was significantly higher in GA $(0.82 \pm 0.001 \mathrm{~mm})$ and in SC (0.71 \pm $0.004 \mathrm{~mm})$ than in NC $(0.68 \pm 0.001 \mathrm{~mm})$ (Table 1$)(P<$ $0.0001)$. Small planktonic copepods $(<1.45 \mathrm{~mm})$ contributed to $100,99.9$ and $98 \%$ of total copepod abundance in SC, NC and GA, respectively, while the largest copepods (1.45$2.5 \mathrm{~mm}$ ) represented mostly by one species Aglaodiaptomus leptopus (Forbes, 1882) (1.6 mm) did not exceed $2 \%$ at NC and GA (Table 2).

The classification of copepod species according to their Indicator Value (IndVal) in these three coastal marine ecosystems under contrasting anthropogenic inputs showed that each zone was characterized by a suitable and specific environmental health indicator. In fact, in the SC, several species have a high IndVal $(>40 \%)$. They were composed of 10 copepod species which represented $45 \%$ of the copepod species' richness in this area and in particular Oithona nana (Indval = $92 \%)$ which was the most indicative of water quality in this zone, followed by Eucalanus sp. (Dana, 1852) (87\%) and Oithona similis (77\%) (Table 3). Concerning the NC, IndVal was very low and only Tisbe battagliai (40\%) and Harpacticus littoralis (Sars, 1910) (39\%) could be good indicators. However, in GA the best indicator species are Euterpina acutifrons (64\%), Temora stylifera (Dana, 1849) (58\%) and Paracalanus parvus (47\%) (Table 3).

\section{Multivariate analysis}

The Canonical Correspondence Analysis (CCA) on the zooplankton parameters and various physico-chemical and biogeochemical factors explained $46.5 \%$ for the $\mathrm{F}_{1}$ and $\mathrm{F}_{2}$ axes (Figure 6). The F1 axis (26.9\%), selected positively SC stations $(1-10)$ with calanoid, cyclopoid, total copepod, non-copepodzooplankton, total zooplankton and temperature. We note that temperature displayed significant positive correlations with total zooplankton $(r=0.59, P<0.05)$, total copepods $(r=0.60, P<0.05)$ and cyclopoids $(r=0.57, P<0.05)$. $\mathrm{F}_{1}$ axis selected negatively NC stations $(11-20)$ with Evenness index $(J)$, the diversity index $\left(H^{\prime}\right), \mathrm{pH}$, sex ratio and salinity. The $\mathrm{F}_{2}$ axis (19.6\%) selected negatively the GA stations $(21-30)$ with harpacticoid, T-P, $\mathrm{PO}_{4}^{3-}$ and SPM (Figure 6A). The plots of the copepod species confirmed our observation with SC characterized by Oithona nana and Oithona similis among cyclopoid copepods, and GA characterized by Paracalanus parvus among calanoid copepods and Euterpina acutifrons among harpacticoid copepods (Figure 6B).

\section{DISCUSSIDN}

Zooplankton is recognized among the best indicators for investigating and documenting environmental changes (Siokou-Frangou \& Papathanassiou, 1991; Sipkay et al., 

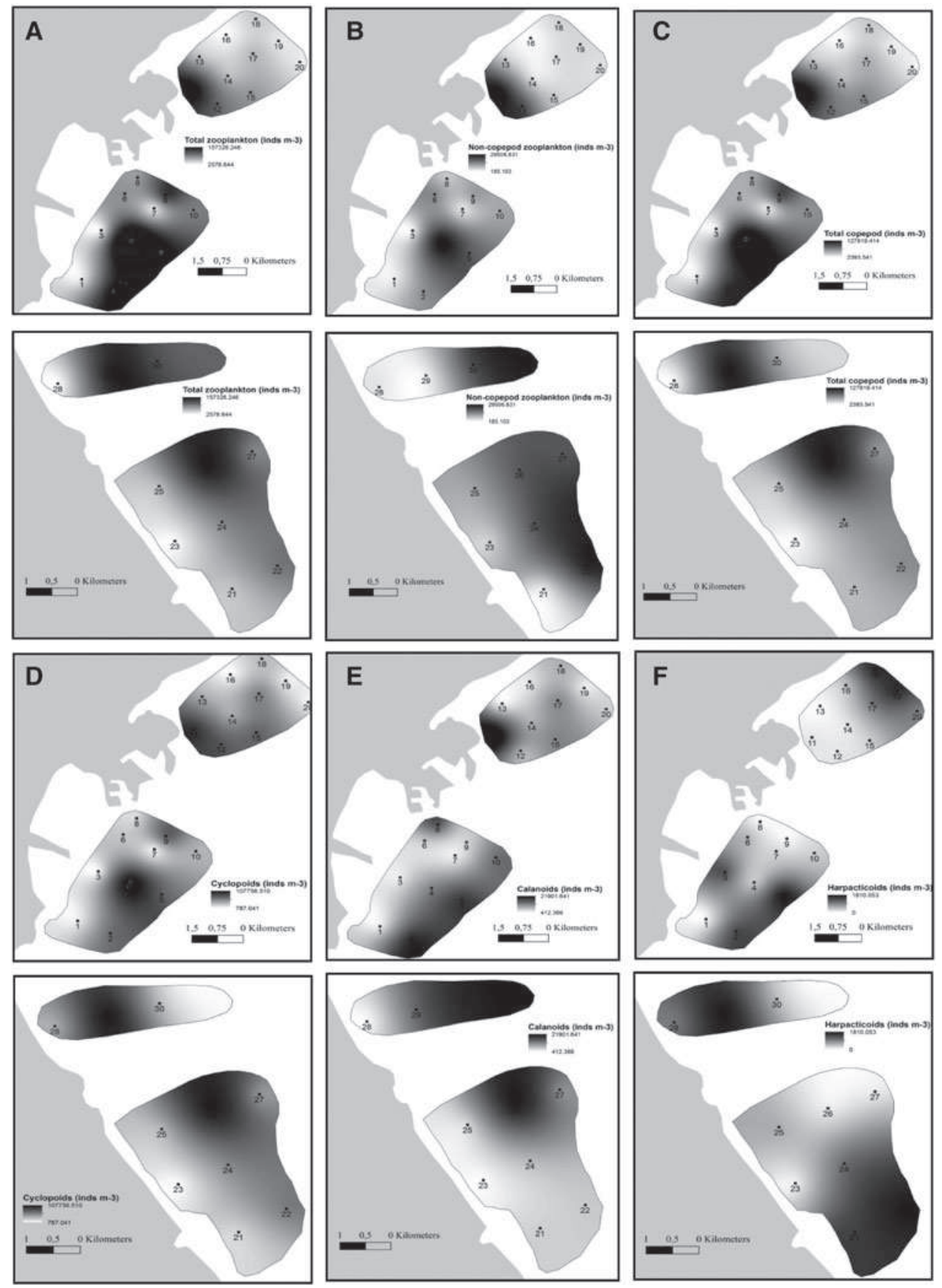

Fig. 4. Spatial variation of zooplankton parameters, i.e. abundance of total zooplankton (A), non-copepod zooplankton (B), total copepods (C), cyclopoids (D), calanoids (E) and harpacticoids (F) in stations sampled in the northern and southern coastal areas of Sfax and the Ghannouch area during autumn (OctoberNovember 2014).

2009; Bagheri et al., 2013). The variations of zooplankton populations are closely related to environmental parameters such as temperature, $\mathrm{pH}$ and salinity (Pascual \& Guichard, 2005; Rossi \& Jamet, 2009; Srichandan et al., 2013). Main zooplankton taxa have short life cycles and their community structure is able to reflect real-time scenarios as it is less enforced by the stability of individuals from previous years (Richardson, 2008; Bagheri et al., 2013). Thus, hypoxic/ anoxic conditions related to organic enrichment are found to be associated with the decrease of zooplankton abundance in eutrophic and/or organically polluted systems (Stalder \& Marcus, 1997; Park \& Marshall, 2000; Gordina et al., 2001) and high turbidity can increase the death rate of copepods (e.g. Castel \& Feurtet, 1992).

\section{Anthropogenic inputs status of the coastal zone}

We found quite high $\mathrm{PO}_{4}^{3-}$ concentrations, very probably related to inputs from the phosphate processing industries (SIAPE-Sfax and GCT-Gabes), revealing different anthropogenic input degrees. In this context, we may say that GA is the most affected by $\mathrm{PO}_{4}^{3-}$ followed by $\mathrm{SC}$ and $\mathrm{NC}$. The 

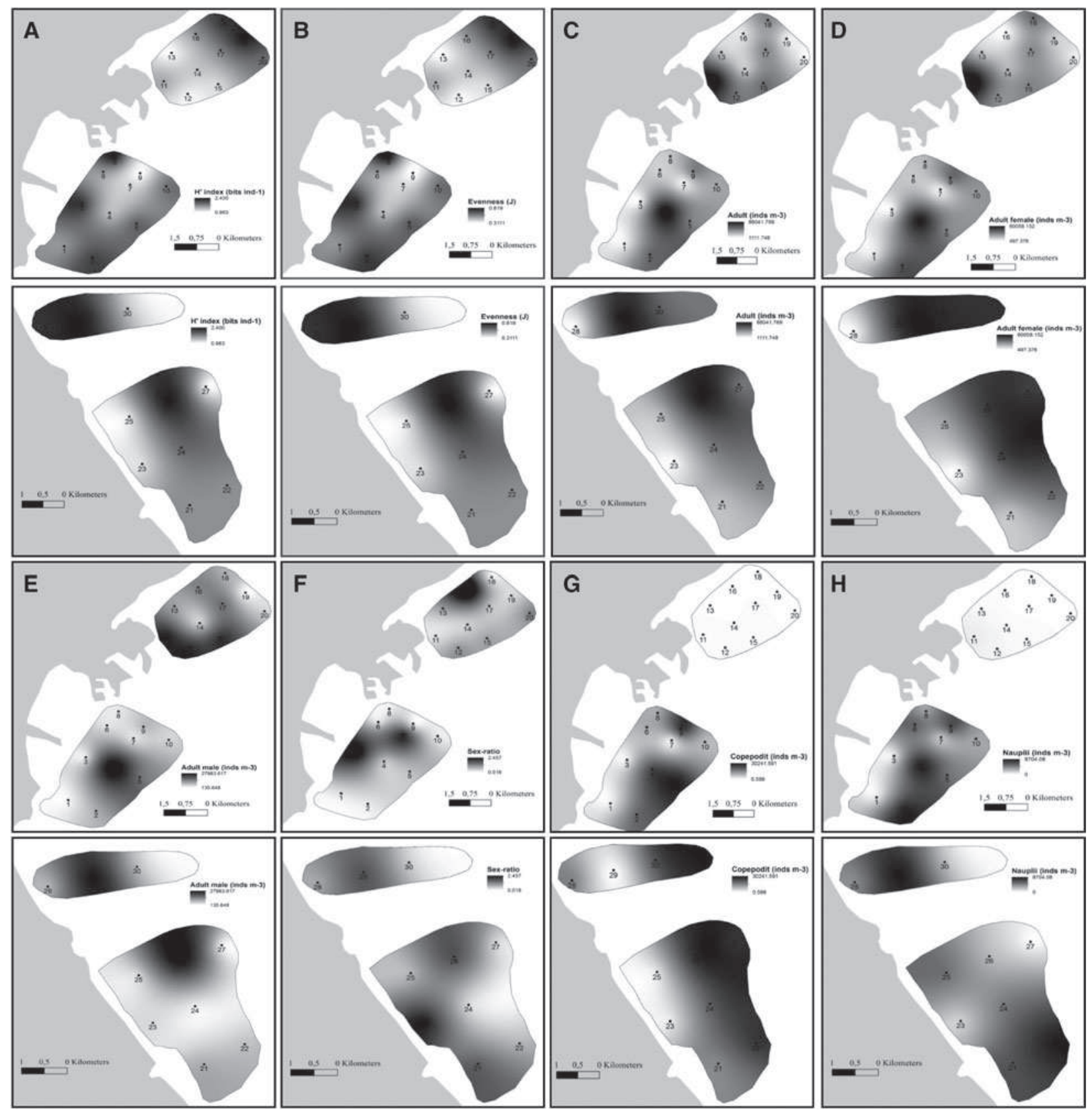

Fig. 5. Spatial variation of zooplankton parameters, i.e. Shannon index $(E)(A)$, Evenness $(J)(B)$, abundance of adult (C), adult female (D), adult male (E), sex-ratio $(\mathrm{F})$, copepodit $(\mathrm{G})$ and nauplii $(\mathrm{H})$ in stations sampled in the northern and southern coastal areas of Sfax and the Ghannouch area during autumn (October-November 2014).

high concentration of $\mathrm{PO}_{4}^{3-}(4.46 \pm 2.60 \mu \mathrm{m})$ in GA was in the range of values previously reported by Baccar (2014) in the Gabes area $(3.73 \pm 1.57 \mu \mathrm{m})$, while the values we found at SC $(3.11 \pm 2.81 \mu \mathrm{m})$ and $\mathrm{NC}(2.07 \pm 0.62 \mu \mathrm{m})$ were far higher than the ones noted by Rekik et al. (2012) (0.28 \pm $0.05 \mu \mathrm{m})$ in SC or by Drira et al. (2009) in the offshore waters of the Gulf of Gabes $(0.06 \pm 0.03 \mu \mathrm{m})$. The highest levels of $\mathrm{PO}_{4}^{3-}$ were always found near the potential sources, i.e. the SIAPE-Sfax plant (station 4), wadi El Maou (station 7), the Sfax fishing harbour (station 9) and the commercial harbour (stations 25 and 27) and the GCT-Gabes phosphoric acid industry (station 28) for GA. Therefore, the high apparent availability of inorganic phosphates in the SC and GA areas were related to the release of phosphate residues from the SIAPE and GCT-Gabes industries (Bejaoui et al., 2004; Ben Brahim et al., 2010; Rekik et al., 2012; Ben Salem et al., 2015; Drira et al., 2016). Ammonium, which was the chemical factor with the most significant differences between areas, showed the highest value in the SC (ANOVA, $P<0.001$ ). The importance of ammonium compared with nitrite and nitrate was a typical finding of coastal eutrophic waters due to anthropogenic pollution, mainly represented by untreated discharges (Nuccio et al., 2003; Bouchouicha-Smida et al., 2012). Our result also showed that NC was slightly alkaline $(\mathrm{pH} 8.12 \pm 0.06)$ compared with GA $(\mathrm{pH}=8.05 \pm 0.07)$, and SC $(\mathrm{pH}=7.99 \pm 0.07)$, which is in agreement with results reported by Rekik et al. (2013) at the same season $(8.16 \pm 0.3)$ for this area. 
Table 3. Classification of copepod species according to their Indicator Value (IndVal) in each zone i.e. the northern and southern coastal areas of Sfax and the Ghannouch area sampled in October-November 2014.

\begin{tabular}{|c|c|c|c|c|c|}
\hline \multicolumn{2}{|l|}{ Southern coastal area } & \multicolumn{2}{|l|}{ Northern coastal area } & \multicolumn{2}{|l|}{ Ghannouch area } \\
\hline Copepod species & $\begin{array}{c}\text { IndVal } \\
(\%)\end{array}$ & Copepod species & $\begin{array}{c}\text { IndVal } \\
(\%)\end{array}$ & Copepod species & $\begin{array}{c}\text { IndVal } \\
(\%)\end{array}$ \\
\hline Oithona nana (Giesbrecht, 1892) & 92.2 & Tisbe battagliai (Volkmann-Rocco, 1972) & 40.0 & Euterpina acutifrons (Dana, 1847) & $64 \cdot 3$ \\
\hline Eucalanus sp. (Dana, 1852) & 86.9 & Harpacticus littoralis (Sars, 1910) & 39.0 & Temora stylifera (Dana, 1849) & 58.2 \\
\hline Oithona similis (Claus, 1866) & 77.2 & Clytemnestra scutellata (Dana, 1847) & 28.3 & Paracalanus parvus (Claus, 1863) & 46.6 \\
\hline Acartia clausi (Giesbrecht, 1889) & 75.7 & Paracartia grani (Sars, 1904) & 28.2 & Aglaodiaptomus leptopus (Forbes, 1882) & 38.7 \\
\hline Centropages kröyeri (Giesbrecht, 1893) & 72.0 & Tigriopus sp. (Mori, 1932) & 20.0 & Temora longicornis (Müller, 1792) & 28.7 \\
\hline Oithona plumifera (Baird, 1843) & 70.2 & Acanthocyclops vernalis (Fischer, 1853) & 15.8 & Oithona plumifera (Baird, 1843) & 16.5 \\
\hline Temora sp. (Baird, 1850) & 63.9 & Oithona plumifera (Baird, 1843) & 9.1 & Oithona similis (Claus, 1866) & 15.4 \\
\hline Paracartia grani (Sars, 1904) & 51.7 & Centropages kröyeri (Giesbrecht, 1893) & 8.8 & Acartia discaudata (Giesbrecht, 1882) & 9.2 \\
\hline Acartia sp. (Dana, 1846) & 42.7 & Eucalanus sp. (Dana, 1852) & 8.4 & Paracartia grani (Sars, 1904) & 8.6 \\
\hline Paracalanus parvus (Claus, 1863) & 40.6 & Paracalanus parvus (Claus, 1863) & 8.3 & Acartia sp. (Dana, 1846) & 5.9 \\
\hline Oithona sp. (Baird, 1843) & 38.5 & Oithona similis (Claus, 1866) & 7.4 & Oithona nana (Giesbrecht, 1892) & 4.4 \\
\hline Euterpina acutifrons (Dana, 1847) & 24.8 & Temora longicornis (Müller, 1792) & 6.4 & Temora sp. (Baird, 1850) & 2.9 \\
\hline Clytemnestra scutellata (Dana, 1847) & 23.8 & Microsettela norvegica (Boeck, 1865) & 4.1 & Centropages kröyeri (Giesbrecht, 1893) & 2.7 \\
\hline Acanthocyclops vernalis (Fischer, 1853) & 20.5 & Acartia sp. (Dana, 1846) & 2.9 & Eucalanus sp. (Dana, 1852) & 0.5 \\
\hline Harpacticus littoralis (Sars, 1910) & 17.5 & Euterpina acutifrons (Dana, 1847) & 2.8 & & \\
\hline Microsettela norvegica (Boeck, 1865) & 11.8 & Oithona nana (Giesbrecht, 1892) & 2.8 & & \\
\hline Acanthocyclops sp. (Kiefer, 1927) & 10.0 & Acartia clausi (Giesbrecht, 1889) & 2.1 & & \\
\hline Oithona setigera (Crisafi, 1959) & 10.0 & Oithona sp. (Baird, 1843) & 1.1 & & \\
\hline Paracartia latisetosa (Kritchagin, 1873) & 10.0 & Aglaodiaptomus leptopus (Forbes, 1882) & 0.6 & & \\
\hline Temora longicornis (Müller, 1792) & 7.1 & Temora stylifera (Dana, 1849) & 0.6 & & \\
\hline Acartia discaudata (Giesbrecht, 1882) & 0.8 & & & & \\
\hline Aglaodiaptomus leptopus (Forbes, 1882) & 0.0 & & & & \\
\hline
\end{tabular}

\section{Copepod assemblages as a bioindicator of environmental quality}

Investigations on zooplankton community in relation with anthropogenic inputs have already been conducted in the Mediterranean Sea (Siokou-Frangou, 1996; Jamet et al., 2001; Isinibilir et al., 2008; Papantoniou et al., 2015). One previous study has been performed so far in the coastal waters of the Gulf of Gabes, i.e. in the SC (Drira et al., submitted). In this study, we tried to determine the effects of anthropogenic inputs on planktonic copepods by comparing the abundance and spatial distribution of the main species in three coastal marine areas with different anthropogenic input levels. Our results showed a clear dominance of copepods (nauplii stage to the adult stage) in the three areas, representing 76,83 and $84 \%$ of total zooplankton in GA, SC and NC, respectively. The dominance of copepods has already been reported in several studies in the Gulf of Gabes: in SC (5-50\%; Ben Salem et al., 2015), NC (82\%, Rekik et al., 2012), in the city of Gabes: Ghannouch and Zarrat (46-83\%; Baccar, 2014) and in offshore waters of the Gulf of Gabes (83\%; Drira et al., 2014; Ben Ltaief et al., 2015).

In this study, we reported the presence of 25 species belonging to 13 families and three orders, namely calanoid, cyclopoid and harpacticoid, while poecilostomatoid were virtually absent. We found a preponderance of cyclopoids, particularly in SC (with $80 \%$ of total copepod abundance), while calanoids were also important in GA (51\%) and NC (43\%). This is consistent with previous works showing that cyclopoids are numerically the most important group in the Gulf of Gabes (Drira et al., 2009, 2014; Ben Ltaief et al., 2015). This predominance of cyclopoids in such polluted areas agrees with their cosmopolitan and less demanding character in terms of environmental conditions compared with other groups (Sarkka et al., 1998). Adult copepods dominated other developmental stages of copepods in the three study sites with adult females being predominant $(>58 \%$ of adults). The dominance of females was already reported in the Gulf of Gabes (2005-2007) (Drira et al., 2010a, b, 2014). Dominance of females compared with males, which reduces the sex ratio (Kiorboe, 2006), may be due to the higher mortality of males because of their increased vulnerability to predation during their search for mates (Mendes-Gusmão et al., 2013). In addition, environmental factors such as pollution have strong effects on copepod sex ratio, and suggest that differential physiological longevity of males and females may be more important in determining the sex ratio (Mendes-Gusmão et al., 2013). Oithonids were numerically very important with $O$. nana (36\% of zooplankton abundance in SC, $12.7 \%$ in NC and $6.4 \%$ in GA and $O$. similis ( $23 \%$ in SC, $21 \%$ in NC and $17 \%$ in GA) as the main species. In previous studies, $O$. nana were also reported at a very high abundance in SC (Drira et al., submitted) as well as in NC before (2007; Rekik et al., 2012) and after (20092010; Rekik et al., 2013) the Taparura restoration process. In the CCA analysis, Oithonids (and O. similis) were negatively correlated with the NC stations corresponding to the less disturbed area and positively correlated with the polluted SC stations, which clearly indicates an affinity of these copepods for anthropogenic inputs. In general, oithonidae may survive in a wide range of habitats and maintain their populations under adverse conditions because they are morphologically less specialized than calanoids (Paffenhöfer, 1993). In agreement with our study, Oithonidae, and more specifically O. nana, are often associated with a high degree of anthropogenic inputs and regarded as a bio-indicator species of anthropogenic pollution (Annabi-Trabelsi et al., 2005; Drira et al., 2014; Serranito et al., 2016). The good adaptation of 


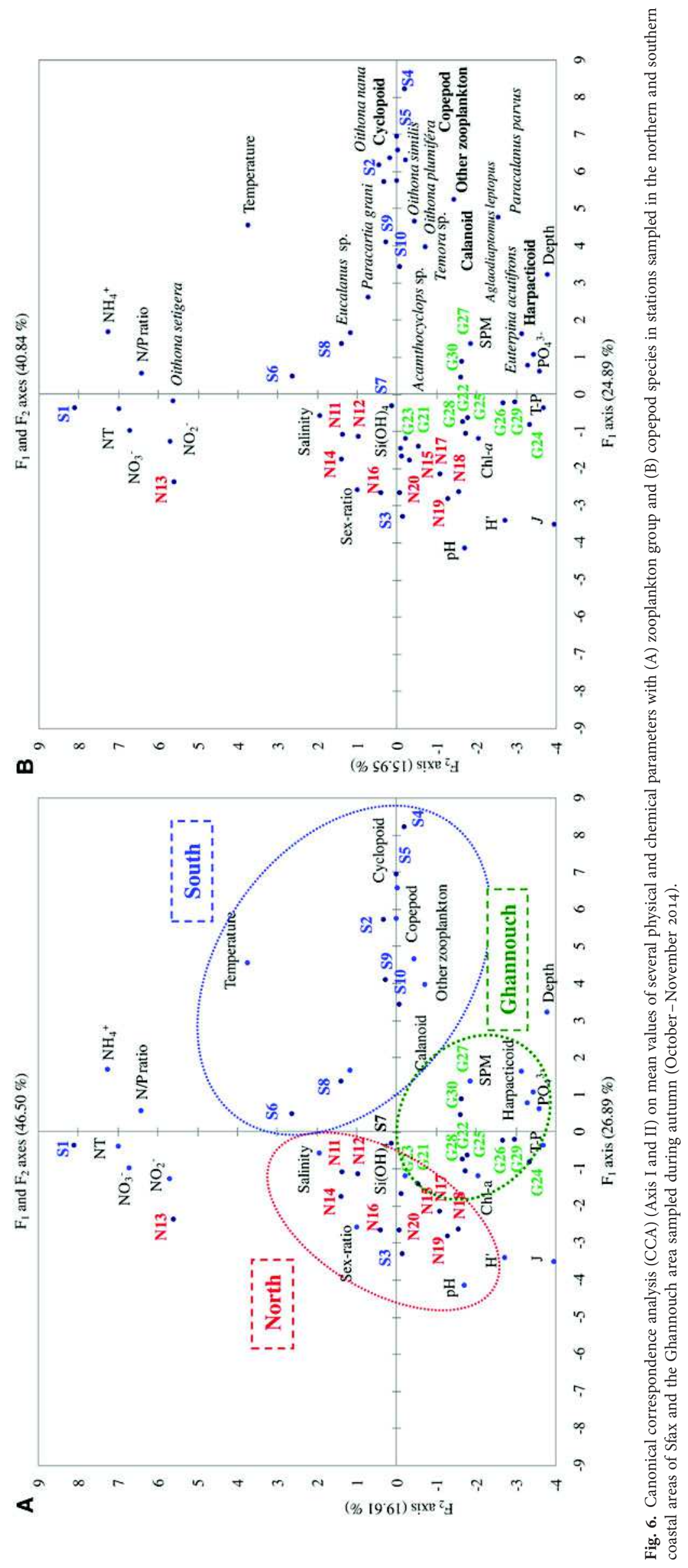


O. nana to anthropized areas can be partly explained by its feeding habits (Serranito et al., 2016). It has been reported to be more flexible in its diet compared with other copepods, thus able to adapt to a wide range of food resources (Moraitou-Apostolopoulou, 1976; Lampitt \& Gamble, 1982; Rekik et al., 2012; Serranito et al., 2016) and having a mixotrophic diet by incorporating faecal matter, ciliates protozoa and dinoflagellates (Williams \& Muxagata, 2006). This small euryoecious species is characterized by a high tolerance to various environmental parameters (Riccardi \& Mariotto, 2000). Its shorter life cycle and higher reproduction rate compared with larger copepods could also partly explain its higher success in adapting to new conditions (Gallienne \& Robins, 2001). Oithona similis is a ubiquitous and abundant cyclopoid not only in our study site, but also in the Algerian basin (Riandey et al., 2005), in the Bay of Tunis (Daly-Yahia et al., 2004), in the lagoon of Tunis (Annabi-Trabelsi et al., 2005) and in the offshore waters of the Gulf of Gabes (Drira et al., 2009). In our study, harpacticoid and more specifically Euterpina acutifrons, were clearly associated to highly polluted conditions as they were correlated to GA stations characterized by the highest SPM and $\mathrm{PO}_{4}^{3-}$ concentrations. Euterpina acutifrons, known as eurythermic and euryhaline, lives in marine coastal areas (Furnestin, 1960; Moreira et al., 1982; Delia Vinas et al., 2010). The plasticity of this species which occupies several coastal habitats is often due to its broad trophic spectrum (phytoplankton, microplankton and detritus) (Goswani, 1976; Moreira et al., 1983).

The taxonomic diversity is also strongly influenced by anthropogenic inputs (Danilov \& Ekelund, 1999). In the present work, we showed that NC, considered as a restored environment in term of phosphogypsum contamination, was characterized by a taxonomic diversity $\left(\mathrm{H}^{\prime}=1.95\right.$ bits ind $^{-1}$, $\left.\mathrm{J}^{\prime}=0.6\right)$ higher than in $\mathrm{GA}\left(\mathrm{H}^{\prime}=1.74\right.$ bits ind $\left.^{-1}, \mathrm{~J}^{\prime}=0.6\right)$ and SC $\left(\mathrm{H}^{\prime}=1.49\right.$ bits ind $\left.^{-1}, \mathrm{~J}^{\prime}=0.5\right)$. Taxonomic diversity (as Piélou's evenness) as well as Oithonidae relative abundance were singled out as the most pertinent indicators of anthropogenic pollution in the case study of the Bay of Toulon (Mediterranean Sea) (Serranito et al., 2016). In our study, based on the same indicators we could classify the three study sites according to the significance of pollution impact on copepods as follows: $\mathrm{NC}<\mathrm{GA}<\mathrm{SC}$ with the diversity $\left(\mathrm{H}^{\prime}=1.95,1.74\right.$ and 1.49 bits $^{-1} \mathrm{ind}^{-1}$, respectively), and GA $<\mathrm{NC}<\mathrm{SC}$ with the percentage of oithonidae (43, 51 and $79 \%$, respectively). We can also note that the mean Indicator Values for Oithonidae are also higher for the SC area (48\%) than for NC and GA (3.7 and $12.2 \%$ respectively), which is consistent with the indicator based on this copepod family. In this context, we may assume that GA, although the most affected by orthophosphates $(4.46 \pm 2.60 \mu \mathrm{m})$ is a more pollution-resistant ecosystem than SC (3.11 \pm $2.81 \mu \mathrm{m})$ compared with $\mathrm{NC}(2.07 \pm 0.62 \mu \mathrm{m})$.

\section{CONCLUSION}

This study was undertaken to assess the zooplankton communities in accordance with anthropogenic inputs in the Sfax northern and southern coasts and in the Ghannouch area during October and November 2014. These three areas were characterized by different degrees of anthropogenic inputs characterized by levels of $\mathrm{PO}_{4}^{3-}$ with highest values at GA (stations 25 and 27 near the commercial harbour and station 28 in front of GCT-Gabes) and lowest at NC. The most abundant species in the three environments were O. nana, O. similis and Paracalanus parvus while two species were reported for the first time in the Gulf of Gabes (Aglaodiaptomus leptopus and Eucalanus sp.). Oithona nana and O. similis could be used as an indicator of anthropogenic inputs in the Gulf of Gabes. Our results indicate that the fluctuation of copepod abundances may be a useful tool to evaluate the ecosystem health status. The present work shows that the Northern coast, considered as a restored and reclaimed environment, is characterized by slightly higher species diversity, while the Ghannouch area, although the most affected by orthophosphates, was found to be more pollution-resistant than the southern coast. Meanwhile, our study can be useful in the management of this ecosystem for planning the best disposal options for treating urban and industrial wastes in the gulf s coastal waters.

\section{ACKNDWLEDGEMENTS}

This work was conducted in the Biodiversity and Aquatic Ecosystems UR/11ES72 Research Unit at the University of Sfax in collaboration with the Mediterranean Institute of Oceanography (MIO) Marseille, France. This work was carried out in the framework of the IRD Action South project 'MANGA' and the IRD French-Tunisian International Joint Laboratory (LMI) 'COSYS-Med' This work is a contribution of the $\mathrm{WP}_{3} \mathrm{C}_{3} \mathrm{~A}$-Action MERMEX/ MISTRALS. This study was carried out in the framework of the postdoctoral fellowship of Z. Drira (University of Sfax, Tunisia). The authors acknowledge $\mathrm{Mr} \mathrm{T}$. Omar and $\mathrm{Mr}$ H. Sahnoun for their technical help during the cruise. We thank the core parameter analytical platform (PAPB) of the MIO for performing analyses of chl- $a$, Phaeo- $a$, POC and PON. Special thanks are also due to Mr K. Maaloul, translator and English professor at the Sfax Faculty of Sciences, University of Sfax (Sfax, Tunisia) for constructive proofreading and language improving services.

\section{REFERENCES}

Aloulou F., Elleuch B. and Kallel M. (2012) Benthic foraminiferal assemblages as pollution proxies in the northern coast of Gabes Gulf, Tunisia. Environmental Monitoring and Assessment 184, 777-795.

Annabi-Trabelsi N., Daly-Yahia M.N., Romdhane M.S. and Ben-Maïz N. (2005) Seasonal variability of planktonic copepods in Tunis north lagoon (Tunisia, North Africa). Cahiers de Biologie Marine 46, 325-333.

APHA (1992) American public health association standard methods for the examination of water and wastewater. Washington, DC: American Public Health Association.

Baccar A. (2014) Contribution à l'étude écologique du zooplancton de la côte du golfe de Gabès. Mastère. Université de Gabès Faculté des sciences de Gabès Tunisie.

Bagheri S., Sabkara J., Mirzajani A., Khodaparast S.H., Yosefzad E. and Yeok F.S. (2013) List of zooplankton taxa in the Caspian sea waters of Iran. Journal of Marine Biology 2013, 1-7.

Bahloul M., Chabbi I., Dammak R., Amdouni R., Medhioub K. and Azri C. (2015) Geochemical behaviour of $\mathrm{PM}_{10}$ aerosol constituents under the influence of succeeding anticyclonic/cyclonic situations: 
case of Sfax City, southern Tunisia. Environmental Monitoring and Assessment 187, 757.

Bahri-Trabelsi E.L., Armi Z., Trabelsi-Annabi N., Shili A. and Ben Maiz N. (2013) Water quality variables as indicators in the restoration impact assessment of the north lagoon of Tunis, South Mediterranean. Journal of Sea Research 79, 12-19.

Barhoumi S., Messaoudi I., Deli T., Saïd K. and Kerkeni A. (2009) Cadmium bioaccumulation in three benthic fish species, Salaria basilisca, Zosterisessor ophiocephalus and Solea vulgaris collected from the Gulf of Gabes in Tunisia. Journal of Environmental Sciences 21, 980-984.

Beaugrand G., Edwards M. and Legendre L. (2010) Marine biodiversity, ecosystem functioning, and carbon cycles. Proceedings of the National Academy of Sciences USA 107, 10120-10124.

Bejaoui B., Raïs S. and Koutitonsky V. (2004) Modelisation de la dispersion du phosphogypse dans le Golfe de Gabes. Bulletin de l'Institut National des Sciences et Technologie de la Mer Salammbô 31, 103-109.

Ben Brahim M., Hamza A., Hannachi I., Rebai A., Jarboui O., Bouain A. and Aleya L. (2010) Variability in the structure of epiphytic assemblages of Posidonia oceanica in relation to human interferences in the Gulf of Gabes, Tunisia. Marine Environmental Research 70, 411-421.

Ben Ltaief T., Drira Z., Devenon J.L., Hamza A., Ayadia H. and Pagano M. (2017) How could thermal stratification affect horizontal distribution of depth-integrated metazooplankton communities in the Gulf of Gabes (Tunisia)? Marine Biology Research. 13, 269-287.

Ben Ltaief T., Drira Z., Hannachi I., Bel Hassen M., Hamza A., Pagano M. and Ayadi H. (2015) What are the factors leading to the success of small planktonic copepods in the Gulf of Gabes, Tunisia? Journal of the Marine Biological Association of the United Kingdom 95, 747-761.

Ben Salem Z. and Ayadi H. (2016) Heavy metal accumulation in Diplodus annularis, Liza aurata, and Solea vulgaris relevant to their concentration in water and sediment from the southwestern Mediterranean (coast of Sfax). Environmental Science and Pollution Research 23, 13895-13906.

Ben Salem Z., Drira Z. and Ayadi H. (2015) What factors drive the variations of phytoplankton, ciliate and mesozooplankton communities in the polluted southern coast of Sfax, Tunisia? Environmental Science and Pollution Research 22, 11764-11780.

Bouchouicha-Smida D., Sahraoui I., Mabrouk H.H. and Sakka Hlaili A. (2012) Seasonal dynamics of genus Alexandrium (potentially toxic dinoflagellate) in the lagoon of Bizerte (North of Tunisia) and controls by the abiotic factors. Comptes Rendus Biologies 335, 406-416.

Bradford-Grieve J.M. (1999) Copepoda. Sub-order: Calanoida, family: Acartiidae, genera: Acartia, Paracartia, Pteriacartia. Copenhagen: ICES Identification Leaflets for Plankton.

Brander K. (2010) Impacts of climate change on fisheries. Journal of Marine System 79, 389-402.

Burrows M.T., Schoeman D.S., Buckley L.B., Moore P., Poloczanska E.S., Brander K.M., Brown C., Bruno J.F., Duarte C.M., Halpern B.S., Holding J., Kappel C.V., Kiessling W., O'Connor M.I., Pandolfi J.M., Parmesan C., Schwing F.B., Sydeman W.J. and Richardson A.J. (2011) The pace of shifting climate in marine and terrestrial ecosystems. Science 334, 652-655.

Callaert B., Van Den Bogaert J., Pieters A., Pynaert K., Tison P., Levrau K., Vander Heyde D. and Glaser D. (2009) Taparura project: sustainable coastal development, including the decontamination and rehabilitation of the coastal area of the city of Sfax, Tunisia. Coastal and Maritime Mediterranean Conference 1, 175-178.

Carr M.H., Neigel J.E., Estes J.A., Andelman S., Warner R.R. and Largier J.L. (2003) Comparing marine and terrestrial ecosystems: implications for the design of coastal marine reserves. Ecological Applications 13, 90-107.

Castel J. and Feurtet A. (1992) Fecundity and mortality rates of the copepod Eurytemora affinis hirundoides in a turbid estuary. Marine Biology 107, 119-128.

Cloern J.E. (2001) Our evolving conceptual model of the coastal eutrophication problem. Marine Ecology Progress Series 210, 223-253.

Costanzo G., Campolmi M. and Zagani G. (2007) Stephos marsalensis new species (Copepoda, Calanoida, Stephidae) from coastal waters of Sicily, Italy. Journal of Plankton Research 22, 2007-2014.

Daly-Yahia M.N., Souissi O.S. and Daly-Yahia Kéfi O. (2004) Spatial and temporal structure of planktonic copepods in the Bay of Tunis (Southwestern Mediterranean Sea). Zoological Studies 43, 366-375.

Danilov R.A. and Ekelund N.G.A. (1999) The efficiency of seven diversity and one similarity indices based on phytoplankton data for assessing the level of eutrophication in lakes in central Sweden. Science of the Total Environment 234, 15-23.

Delia Vinas M., Diovisalvi N.R. and Cepeda G.D. (2010) Individual biovolume of some dominant copepod species in coastal waters off Buenos Aires province, Argentine Sea. Brazilian Journal of Oceanography 58, 177-181.

De-Young B., Heath M., Werner F., Megrey B. and Monfray P. (2004) Challenges of modeling ocean basin ecosystems. Science 304, $1463-1466$.

DGPA (2004) Annuaires des statistiques des produits de la pêche. Tunisie: Ministère de l'Agriculture.

Drira Z., Bel Hassen M., Ayadi H. and Aleya L. (2014) What factors drive copepod community dynamics in the Gulf of Gabes, Eastern Mediterranean Sea? Environmental Science and Pollution Research 21, 2918-2934.

Drira Z., Bel Hassen M., Ayadi H., Hamza A., Zarrad R., Bouaïn A. and Aleya L. (2010a) Copepod community structure related to environmental factors from a summer cruise in the Gulf of Gabes (Tunisia, eastern Mediterranean Sea). Journal of the Marine Biological Association of the United Kingdom 90, 145-157.

Drira Z., Bel Hassen M., Hamza A., Rebai A., Bouain A., Ayadi H. and Aleya L. (2009) Spatial and temporal variations of microphytoplankton composition related to hydrographic conditions in the Gulf of Gabes. Journal of the Marine Biological Association of the United Kingdom 89, 1559-1569.

Drira Z., Hamza A., Bel Hassen M., Ayadi H., Bouaïn A. and Aleya L. (2008) Dynamics of dinoflagellates and environmental factors during the summer in the Gulf of Gabes (Tunisia, Eastern Mediterranean Sea). Scientia Marina 72, 59-71.

Drira Z., Hamza A., Bel Hassen M., Ayadi H., Bouaïn A. and Aleya L. (2010b) Coupling of phytoplankton community structure to nutrients, ciliates and copepods in the Gulf of Gabes (south Ionian Sea, Tunisia). Journal of the Marine Biological Association of the United Kingdom 90, 1203-1215.

Drira Z., Kmiha-Megdiche S., Sahnoun H., Hammami A., Allouche N., Tedetti M. and Ayadi H. (2016) Assessment of anthropogenic inputs in the surface waters of the southern coastal area of Sfax during Spring (Tunisia, Southern Mediterranean Sea). Marine Pollution Bulletin 104, $355-363$.

Drira Z., Sahnoun H. and Ayadi H. (2017) Spatial distribution and source identification of heavy metals in surface waters of three coastal areas (Gulf of Gabes, Tunisia). Polish Journal of Environmental Studies 26, 1-13.

Dufrene M. and Legendre P. (1997) Species assemblages and indicator species: the need for a flexible asymmetrical approach. Ecological Monographs 67, 345-366. 
Feki W., Hamza A., Frossard V., Abdennadher M., Hannachi I., Jacquot M., Bel Hassen M. and Aleya L. (2013) What are the potential drivers of blooms of the toxic dinoflagellate Karenia selliformis? A 10-year study in the Gulf of Gabes, Tunisia, southwestern Mediterranean Sea. Harmful Algae 23, 8-18.

Furnestin M.L. (1960) Zooplancton du Golfe du Lion et de la côte orientale de Corse. Revue des Travaux de l'Institut des Pêches Maritimes 24, $153-252$.

Gallienne C.P. and Robins D.B. (2001) Is Oithona the most important copepod in the world's oceans? Journal of Plankton Research 23, $1421-1432$.

Gargouri D., Azri C., Serbaji M.M., Jedoui Y. and Montacer M. (2011) Heavy metal concentrations in the surface marine sediments of Sfax Coast, Tunisia. Environmental Monitoring and Assessment 175, 519-530.

Gargouri D., Bahloul M. and Chafai A. (2015) Sediment quality and potential toxicity assessment in two open/semi-closed Mediterranean sea areas: a case study of Sfax coast (Tunisia). Water Environment Research 87, 470-479.

Gargouri T. (2006) Diagnostique de la zone côtière Sud de Grand Sfax, Projet SMAP III- Tunisie (2006-2008). Tunisie: Stratégie de Gestion Intégré de la zone Côtière Sud de Grand Sfax.

Gargouri-Ben Ayed Z., Souissi R., Soussi M., Abdeljaouad S. and Zouari K. (2007) Sedimentary dynamics and ecological state of Nakta Tidal Flat (Littoral), South of Sfax, Gulf of Gabes, Tunisia. Chinese Journal of Geochemistry 26, 244-251.

Gordina A.D., Pavlova E.V., Ovsyany E.I., Wilson J.G., Kemp R.B. and Romanov A.S. (2001) Long-term changes in Sevastopol Bay (the Black Sea) with particular reference the ichthyoplankton and zooplankton. Estuarine, Coastal and Shelf Science 52, 1-13.

Goswani S.C. (1976) Larval stages of laboratory reared harpacticoid copepod Euterpina acutifrons (Dana). National Institute of Oceanography 8, 123-132.

Hamza-Chaffai A., Amiard-Triquet C. and El Abed A. (1997) Metallothionein-like protein, is it an efficient biomarker of metal contamination? A case study based on fish from the Tunisian coast. Archives of Environmental Contamination and Toxicology 33, 53-62.

Hamza-Chaffai A., Cosson R.P., Amiard-Triquet C. and El Abed A. (1995) Physico-chemical forms of storage of metals (Cd, Cu and $\mathrm{Zn}$ ) and metallothionein-like proteins in gills and liver of marine fish from the Tunisian coast: ecotoxicological consequences. Comparative Biochemistry and Physiology Part C: Pharmacology, Toxicology and Endocrinology 111, 329-341.

Hamza-Chaffai A., Pellerin J. and Amiard J.C. (2003) Health assessment of a marine bivalve Ruditapes decussatus from the Gulf of Gabes (Tunisia). Environment International 28, 609-617.

Hays G.C., Richardson A.J. and Robinson C. (2005) Climate change and marine plankton. Trends in Ecology and Evolution 20, 337-344.

Hemraj D.A., Hossain M.A., Ye Q., Qin J.G. and Leterme S.C. (2017) Plankton bioindicators of environmental conditions in coastal lagoons. Estuarine, Coastal and Shelf Science 184, 102-114.

Isinibilir M., Kideys A.E., Tarkan A.N. and Yilmaz I.N. (2008) Annual cycle of zooplankton abundance and species composition in Izmit Bay (the northeastern Marmara Sea). Estuarine, Coastal and Shelf Science $78,739-747$.

Jamet J., Boge G., Richard S., Geneys C. and Jamet D. (2001) The zooplankton community in bays of Toulon area (northwest Mediterranean Sea, France). Hydrobiologia 457, 155-165.

Kennedy V.S., Twilley R.R., Kleypas J.A., Cowan J.H.J. and Hare S.R. (2002) Coastal and marine ecosystems and global climate change: potential effects on U.S. Arlington, VA: Resources, Pew Center on Global Climate Change.

Kiorboe T. (2006) Sex, sex-ratios and the dynamics of pelagic copepod populations. Oecologia 148, 40-50.

Lampitt R.S. and Gamble J.C. (1982) Diet and respiration of the small planktonic marine copepod Oithona nana. Marine Biology 66, 185-190.

Longhurst A.R. (2007) Ecological geography of the sea. Amsterdam: Elsevier.

Louati A., Elleuch B., Kallel A., Saliot A., Dagaut J. and Oudot J. (2001) Hydrocarbon contamination of coastal sediments from the Sfax area (Tunisia), Mediterranean Sea. Marine Pollution Bulletin 42, 445-452.

Mendes-Gusmão L.F., McKinnon A.D. and Richardson A.J. (2013) No evidence of predation causing female-biased sex ratios in marine pelagic copepods. Marine Ecology Progress Series 482, 279-298.

Moraitou-Apostolopoulou M. (1976) Étude comparative du zooplancton superficiel $(0-100 \mathrm{~cm})$ à une zone hautement polluée et une autre relativement propre (Golfe Saronique - Grèce). Rapport Procès Verbal Réunion de la Commission International pour l'Exploration Scientifique de Méditerranée 23, 59-60.

Moreira G.S., Jillett J.B., Vernberg W.B. and Weinrich M. (1982) The combined effects of temperature and salinity on the survival of Euterpina acutifrons (Dana) (Copepoda, Harpacticoida) from the New Zealand and Brazilian coasts. Journal of Plankton Research 4, $85-91$.

Moreira G.S., Yamashita C. and McNamara J.C. (1983) Seasonal variation in abundance of the developmental stages of Euterpina acutifrons (Copepoda, Harpacticoida) from São Sebastião Channel, Southern Brazil. Marine Biology 74, 111-114.

Neukermans G., Ruddick K., Loisel H. and Roose P. (2012) Optimization and quality control of suspended particulate matter concentration measurement using turbidity measurements. Limnology and Oceanography: Methods 10, 1011-1023.

Newton A., Icely J.D., Falcao M., Nobre A. and Nunes J.P., Ferreira J.G., Vale C. (2003) Evaluation of eutrophication in the Ria Formosa coastal lagoon, Portugal. Continental Shelf Research 23, 1945-1961.

Nuccio C., Melillo C., Massi L. and Innamorati M. (2003) Phytoplankton abundance, community structure and diversity in eutrophycated Orbetello lagoon (Tuscany) from 1995 to 2001. Oceanologica Acta 26, 15-25.

Paffenhöfer G.A. (1993) On the ecology of marine cyclopoid copepods (Crustacea, Copepoda). Journal of Plankton Research 15, 37-55.

Papantoniou G., Danielidis D.B., Spyropoulou A. and Ragopoulu N. (2015) Spatial and temporal variability of small-sized copepod assemblages in a shallow semi-enclosed embayment (Kalloni Gulf, NE Mediterranean Sea). Journal of the Marine Biological Association of the United Kingdom 2, 349-360.

Park G.S. and Marshall H.G. (2000) Estuarine relationships between zooplankton community structure and trophic gradients. Journal of Plankton Research 22, 121-135.

Pascual M. and Guichard F. (2005) Criticality and disturbance in spatial ecological systems. Trends in Ecology and Evolution 20, 88-95.

Pielou E.C. (1966) The measurement of diversity in different types of biological collections. Journal of Theoretical Biology 13, 131-144.

Raimbault P., Diaz F., Pouvesle W. and Boudjellal B. (1999) Simultaneous determination of particulate organic carbon, nitrogen and phosphorus collected on filters, using a semi- automatic wet-oxidation method. Marine Ecology Progress Series 180, 289-295. 
Raimbault P., Lantoine F. and Neveux J. (2004) Dosage rapide de la chlorophylle- $a$ et des phéopigments- $a$ par fluorimétrie après extraction au méthanol. Comparaison avec la méthode classique d'extraction à l'acétone. Océanis 30, 189-205.

Rekik A., Denis M., Maalej S. and Ayadi H. (2015) Spatial and seasonal variability of pico-, nano- and microphytoplankton at the bottom seawater in the north coast of Sfax, Eastern Mediterranean Sea. Environmental Science and Pollution Research 22, 15961-15975.

Rekik A., Drira Z., Guermazi W., Elloumi J., Maalej S., Aleya L. and Ayadi H. (2012) Impacts of an uncontrolled phosphogypsum dumpsite on summer distribution of phytoplankton, copepods and ciliates in relation to abiotic variables along the near-shore of the southwestern Mediterranean coast. Marine Pollution Bulletin 64, 336-346.

Rekik A., Maalej S., Ayadi H. and Aleya L. (2013) Restoration impact of an uncontrolled phosphogypsum dump site on the seasonal distribution of abiotic variables, phytoplankton and zooplankton along the near shore of the south-western Mediterranean coast. Environmental Science and Pollution Research 20, 3718-3734.

Riandey V., Champalbert G., Carlotti F., Taupier-Letage I. and Thibault-Botha D. (2005) Zooplankton distribution related to the hydrodynamic features in the Algerian Basin (Western Mediterranean Sea) in summer 1997. Deep Sea Research Part I: Oceanographic Research Papers 52, 2029-2048.

Riccardi N. and Mariotto L. (2000) Seasonal variations in copepod body length: a comparison between different species in the lagoon of Venice. Aquatic Ecology 34, 243-252.

Richardson A.J. (2008) In hot water: zooplankton and climate change. Journal of Marine Science 65, 279-295.

Rose M. (1933) Copépodes pélagiques. Faune de la France, Volume 26. Paris: Le chevalier.

Rossi N and Jamet J.L. (2009) Structure and succession of plankton communities in two Mediterranean neighbouring coastal ecosystems (Toulon Bay, France) In Martorino L. and Puopolo K. (eds). New Oceanography Research Developments. Nova Science Publishers, 1-14.

Ruttenberg B.I. and Granek E.F. (2011) Bridging the marine-terrestrial disconnect to improve marine coastal zone science and management. Marine Ecology Progress Series 434, 203-212.

Sampey A., Mckinnon A.D., Meekan M.G. and McCormick M.I. (2007) Glimpse into guts: overview of the feeding of larvae of tropical shorefishes. Marine Ecology Progress Series 339, 243-257.

Sarkka J., Levonen L. and Mäkelä J. (1998) Harpacticoid and cyclopoid fauna of ground water and springs in southern Finland. Journal of Marine Systems 15, 155-161.

Serranito B., Aubert A., Stemmann L., Rossi N. and Jamet J.L. (2016) Proposition of indicators of anthropogenic pressure in the Bay of Toulon (Mediterranean Sea) based on zooplankton time-series. Continental Shelf Research 121, 3-12.

Shannon C.E. and Weaver W. (1949) The mathematical theory of communication. Urbana, IL: University of Illinois Press.

Siokou-Frangou I. (1996) Zooplankton annual cycle in a Mediterranean coastal area. Journal of Plankton Research 18, 203-223.
Siokou-Frangou I. and Papathanassiou E. (1991) Differentiation of zooplankton populations in a polluted area. Marine Ecology Progress Series $76,41-51$

Sipkay C., Kiss K.T., Vadadi-Fülöp C. and Hufnagel L. (2009) Trends in research on the possible effects of climate change concerning aquatic ecosystems with special emphasis on the modeling approach. Applied Ecology and Environmental Research 17, 171-198.

Sokal R.R. and Rohlf F.J. (1981) Biometry: the principles and practices of statistics in biological research, 2nd edition. New York, NY: Freeman and Company W.H.

Srichandan S., Panda C.R. and Rout N.C. (2013) Seasonal distribution of zooplankton in Mahanadi estuary (Odisha), East Coast of India: a taxonomical approach. Zoological Research 9, 17-31.

Stalder L.C. and Marcus N.H. (1997) Zooplankton responses to hypoxia: behavioral patterns and survival of three species of calanoid copepods. Marine Biology 127, 599-607.

Tayibi H., Choura M., López F.A., Alguacil F.J. and López-Delgado A. (2009) Environmental impact and management of phosphogypsum. Journal of Environmental Management 90, 2377-2386.

Ter-Braak C.J.F. (1986) Canonical correspondence analysis: a new eigenvector technique for multivariate direct gradient analysis. Ecology 67, $1167-1179$.

Tregouboff G. and Rose M. (1978a) Manuel de Planctologie Méditerranéenne. Tome I. Paris: CNRS.

Tregouboff G. and Rose M. (1978b) Manuel de Planctonologie Méditerranéenne. Tome II. Paris: CNRS.

Williams J.A. and Muxagata E. (2006) The seasonal abundance and production of Oithona nana (Copepoda: Cyclopoida) in Southampton Water. Journal of Plankton Research 28, 1055-1065.

Zaghden H., Kallel M., Louati A., Elleuch B., Oudot J. and Saliot A. (2005) Hydrocarbons in surface sediments from the Sfax coastal zone, (Tunisia) Mediterranean Sea. Marine Pollution Bulletin 50, $287-294$.

Zaouali J. (1993) Little Syrte benthic communities, Gulf of Gabès, Tunisia Results of the survey campaign of July 1990. Preliminary study; biocenosis and recent thanatocenosis. Marine Life 3, 47-6o.

and

Ziadi B., Dhib A., Turki S. and Aleya L. (2015) Factors driving the seasonal distribution of zooplankton in a eutrophicated Mediterranean lagoon. Marine Pollution Bulletin 97, 224-233.

\section{Correspondence should be addressed to:}

Z. Drira

Department of Life Sciences, Sfax Faculty of Sciences,

Biodiversity and Aquatic Ecosystems UR/11ES72

Research Unit, University of Sfax,

Soukra Road Km 3.5. BP 1171 - PO Box 3000 Sfax, Tunisia email: zaherdrira@yahoo.fr 